\title{
Dominantly acting variants in ARF3 have disruptive consequences on Golgi integrity and cause microcephaly recapitulated in zebrafish
}

\section{Giulia Fasano}

Ospedale Pediatrico Bambino Gesù

\section{Valentina Muto}

Ospedale Pediatrico Bambino Gesù

\section{Francesca Clementina Radio}

Genetic and Rare Disease Research Division, Bambino Gesù Children's Hospital IRCCS, Rome, Italy https://orcid.org/0000-0003-1993-8018

\section{Martina Venditti}

Ospedale Pediatrico Bambino Gesù

\section{Alban Ziegler \\ Département de Génétique, CHU d’Angers}

\section{Giovanni Chillemi}

Tuscia University https://orcid.org/0000-0003-3901-6926

\section{Annalisa Vetro}

Pediatric Neurology, Neurogenetics and Neurobiology Unit and Laboratories, Meyer Children's Hospital, University of Florence

Francesca Pantaleoni

https://orcid.org/0000-0003-0765-9281

\section{Simone Pizzi}

Bambino Gesù Children's Hospital

\section{Libenzio Conti}

Ospedale Pediatrico Bambino Gesù, IRCCS, 00146 Rome https://orcid.org/0000-0001-9466-5473

\section{Stefania Petrini}

Bambino Gesù Children's Hospital

\section{Simona Coppola}

Istituto Superiore di Sanità

\section{Alessandro Bruselles}

Istituto Superiore di Sanità https://orcid.org/0000-0002-1556-4998

\section{Ingrid Guarnetti Prandi}

University of Pisa, 56124 Pisa, Italy

Balasubramanian Chandramouli 
Super Computing Applications and Innovation, CINECA

\section{Magalie Barth}

Céline Bris

Département de Génétique, CHU d’Angers

Donatella Milani

Fondazione IRCCS Ca' Granda Ospedale Maggiore Policlinico

\section{Angelo Selicorni}

ASST Lariana

Marina Macchiaiolo

Ospedale Pediatrico Bambino Gesù, IRCCS

Michaela Gonfiantini

Ospedale Pediatrico Bambino Gesù, IRCCS

\section{Andrea Bartuli}

Bambino Gesù Children's Hospital

\section{Renzo Guerrini}

Children's Hospital A. Meyer-University of Florence

\section{Anne Slavotinek}

University of California and San Francisco

\section{Maria lascone}

ASST Papa Giovanni XXIII

\section{Bruno Dallapiccola}

Bambino Gesù Children Hospital

Antonella Lauri ( $\square$ antonella.lauri@opbg.net)

Ospedale Pediatrico Bambino Gesù, IRCCS

\section{Marco Tartaglia}

Ospedale Pediatrico Bambino Gesù https://orcid.org/0000-0001-7736-9672

\section{Article}

Keywords: ARF3, Golgi integrity, zebrafish, de novo missense variants

Posted Date: August 10th, 2021

DOl: https://doi.org/10.21203/rs.3.rs-678090/v1

License: (c) (1) This work is licensed under a Creative Commons Attribution 4.0 International License. Read Full License

Additional Declarations: There is NO Competing Interest. 
Version of Record: A version of this preprint was published at Nature Communications on November 11th, 2022. See the published version at https://doi.org/10.1038/s41467-022-34354-x. 
1 Dominantly acting variants in $A R F 3$ have disruptive consequences on Golgi 2 integrity and cause microcephaly recapitulated in zebrafish

4 Giulia Fasano ${ }^{1,16}$, Valentina Muto ${ }^{1,16}$, Francesca Clementina Radio ${ }^{1,16}$, Martina 5 Venditti $^{1}$, Alban Ziegler ${ }^{2,3}$, Giovanni Chillemi ${ }^{4,5}$, Annalisa Vetro ${ }^{6}$, Francesca 6 Pantaleoni ${ }^{1}$, Simone Pizzi $^{1}$, Libenzio Adrian Conti ${ }^{7}$, Stefania Petrini ${ }^{7}$, Simona 7 Coppola $^{8}$, Alessandro Bruselles ${ }^{9}$, Ingrid Guarnetti Prandi ${ }^{10}$, Balasubramanian 8 Chandramouli ${ }^{11}$, Magalie Barth ${ }^{2,3}$, Céline Bris ${ }^{2,3}$, Donatella Milani ${ }^{12}$, Angelo Selicorni ${ }^{13}$, 9 Marina Macchiaiolo ${ }^{1}$, Michaela V Gonfiantini ${ }^{1}$, Andrea Bartuli ${ }^{1}$, Renzo Guerrini ${ }^{6}$, Anne Slavotinek ${ }^{14}$, Maria lascone ${ }^{15}$, Bruno Dallapiccola ${ }^{1}$, Antonella Lauri ${ }^{1,17 *}$, Marco 11 Tartaglia ${ }^{1,17 *}$

\section{Author information}

${ }^{1}$ Genetics and Rare Diseases Research Division, Ospedale Pediatrico Bambino Gesù, IRCCS, 00146 Rome, Italy.

2Département de Génétique, CHU d'Angers, 49000 Angers, France.

${ }^{3}$ UFR Santé de l'Université d'Angers, INSERM U1083, CNRS UMR6015, MITOVASC, SFR ICAT, F-49000 Angers, France. ${ }^{4}$ Institute of Biomembranes, Bioenergetics and Molecular Biotechnologies, Centro Nazionale delle Ricerche, 70126, Bari, Italy.

$21{ }^{5}$ Department for Innovation in Biological Agro-food and Forest systems (DIBAF), University of 22 Tuscia, 01100 Viterbo, Italy. ${ }^{6}$ Pediatric Neurology, Neurogenetics and Neurobiology Unit and Laboratories, Meyer Children's Hospital, University of Florence, 50139 Florence, Italy 
Dominantly acting variants in ARF3 have disruptive consequences on Golgi integrity and cause microcephaly recapitulated in zebrafish

Fasano, Muto, Radio, et al.

${ }^{7}$ Confocal Microscopy Core Facility, Ospedale Pediatrico Bambino Gesù, IRCCS, 00146 Rome, Italy.

${ }^{8}$ Centro Nazionale Malattie Rare, Istituto Superiore di Sanità, 00161 Rome, Italy.

${ }^{9}$ Department of Oncology and Molecular Medicine, Istituto Superiore di Sanità, 00161 Rome, Italy.

${ }^{10}$ Department of Chemistry and Industrial Chemistry, University of Pisa, 56124 Pisa, Italy

${ }^{11}$ Super Computing Applications and Innovation, CINECA, 40033 Casalecchio di Reno, Italy.

${ }^{12}$ Pediatric Highly Intensive Care Unit, Fondazione IRCCS Ca' Granda Ospedale Maggiore Policlinico, 20122 Milan, Italy.

${ }^{13}$ Mariani Center for Fragile Children Pediatric Unit, Azienda Socio Sanitaria Territoriale Lariana, 22100 Como, Italy.

${ }^{14}$ Department of Pediatrics, Division of Medical Genetics, University of California, San Francisco, San Francisco, CA 94143, USA.

${ }^{15}$ Medical Genetics, ASST Papa Giovanni XXIII, 24127 Bergamo, Italy.

${ }^{16}$ These authors contributed equally to this work

${ }^{17}$ These authors contributed equally to this work

${ }^{*}$ Corresponding authors:

Antonella Lauri

Genetics and Rare Diseases Research Division

Ospedale Pediatrico Bambino Gesù

Viale di San Paolo, 15

00146 Rome, Italy

Email: antonella.lauri@opbg.net

Marco Tartaglia

Genetics and Rare Diseases Research Division

Ospedale Pediatrico Bambino Gesù

Viale di San Paolo, 15

00146 Rome, Italy

Email: marco.tartaglia@opbg.net 
Dominantly acting variants in ARF3 have disruptive consequences on Golgi integrity and cause microcephaly recapitulated in zebrafish

Fasano, Muto, Radio, et al.

\section{7}

58

59

60

61

62

63

64

65

66

67

68

69

70

71

72

73

74

75

76

77

78

79

80

81

82

83

84

85

86

\section{Email Addresses}

Giulia Fasano

Valentina Muto

Francesca Clementina Radio

Martina Venditti

Alban Ziegler

Giovanni Chillemi

Annalisa Vetro

Francesca Pantaleoni

Simone Pizzi

Adrian Libenzio Conti

Stefania Petrini

Simona Coppola

Alessandro Bruselles

Ingrid Guarnetti Prandi

Balasubramanian Chandramouli

Magalie Barth

Céline Bris

Donatella Milani

Angelo Selicorni

Marina Macchiaiolo

Michaela Veronika Gonfiantini.

Andrea Bartuli

Renzo Guerrini

Anne Slavotinek

Maria lascone

Bruno Dallapiccola

Antonella Lauri

Marco Tartaglia giulia.fasano@opbg.net

valentina.muto@opbg.net

fclementina.radio@opbg.net

martina.venditti@opbg.net

alban.ziegler@chu-angers.fr

gchillemi@unitus.it

annalisa.vetro@meyer.it

francesca.pantaleoni@opbg.net

simone.pizzi@opbg.net

libenzioadrian.conti@opbg.net

stefania.petrini@opbg.net

simona.coppola@iss.it

alessandro.bruselles@iss.it

ingrid.prandi@gmail.com

b.chandramouli@cineca.it

mabarth@chu-angers.fr

cebris@chu-angers.fr

donatella.milani@policlinico.mi.it

angelo.selicorni61@gmail.com

marina.macchiaiolo@opbg.net

mveronik.gonfiantini@opbg.net

andrea.bartuli@opbg.net

renzo.guerrini@meyer.it

anne.slavotinek@ucsf.edu

miascone@asst-pg23.it

bruno.dallapiccola@opbg.net

antonella.lauri@opbg.net

marco.tartaglia@opbg.net 
Dominantly acting variants in ARF3 have disruptive consequences on Golgi integrity and cause microcephaly recapitulated in zebrafish

Fasano, Muto, Radio, et al.

\section{Abstract}

88 Vesicle biogenesis, trafficking and signaling via the ER-Golgi network support essential processes during development and their disruption can lead to neurodevelopmental disorders and neurodegeneration. We report that de novo missense variants in ARF3,

91 encoding a small GTPase regulating Golgi structure and function, cause a

92 neurodevelopmental disease showing microcephaly and progressive cortical atrophy, 93 with microsomia and rib anomalies in severely affected subjects, suggesting a 94 pleiotropic effect. All microcephaly-associated variants clustered in the guanine nucleotide binding pocket and perturbed the biochemical behavior of the protein by stabilizing it in a GTP-bound state. Functional analysis proved the disruptive 97 consequences of the variants on Golgi integrity, and brain and body plan formation. Indepth analysis in zebrafish embryos expressing ARF3 mutants traced back the developmental alterations to defective gastrulation cell movements as the earliest detectable effect. Our findings document a role of ARF3 in Golgi homeostasis and

101 demonstrate an obligate dependence for early development. 
Dominantly acting variants in ARF3 have disruptive consequences on Golgi integrity and cause microcephaly recapitulated in zebrafish

Fasano, Muto, Radio, et al.

102

103

\section{Introduction}

The Golgi apparatus (GA) is a polarized, membrane network-built organelle responsible for transporting, modifying, and packaging proteins and lipids into vesicles for their delivery to targeted destinations ${ }^{1,2}$. It is organized as a series of flattened, stacked pouches (known as cisternae) that are held together by matrix proteins and microtubules, and are structured into two major networks, the cis and trans-Golgi compartments, coordinating proper sorting of proteins and lipids received from the endoplasmic reticulum and directing their transport toward the cell membrane ${ }^{3,4}$. GA organization is highly dynamic and undergoes rapid remodeling in response to different physiological and pathological stimuli via various tightly regulated processes involving ribbon disassembly and tubulovesicular conversion as well as repositioning of Golgi stacks $^{5}$. Besides the role of GA in posttranslational modification and sorting of proteins, a large body of studies recently revealed that GA membranes also provide signaling platforms for the regulation of a wide range of cellular processes (e.g., cell polarization, directed migration, stress response, mitosis, and autophagy) orchestrating animal development, suggesting that GA can act as a cell sensor and regulator similarly to other intracellular organelles ${ }^{4}$. Not surprisingly, in the last years, a number of Mendelian disorders have been causally related to the defective or aberrant function of component of the GA-related transport machinery and disrupted GA function and organization, most of which sharing altered neurodevelopment and early-onset neurodegeneration ${ }^{6-8}$. In these disorders, which have collectively been termed as "Golgipathies", recurrent features include microcephaly, central nervous system (CNS) defects (e.g., delayed myelination, cortical atrophy, abnormal corpus callosum, and pontocerebellar hypoplasia) and developmental delay (DD)/intellectual disability (ID) 8,9 . 
Dominantly acting variants in ARF3 have disruptive consequences on Golgi integrity and cause microcephaly recapitulated in zebrafish

Fasano, Muto, Radio, et al.

127 The five members of the ADP-ribosylation factors (ARF) family of small GTPases

128 (ARF1, ARF3-6) regulate key events in vesicular biogenesis, transport and various GA

129 functions, and participate in the control of bidirectional membrane trafficking required

130 for secretion, endocytosis and recycling ${ }^{10-13}$. These proteins bind to guanine

131 nucleotides with high affinity and specificity, and have a slow intrinsic competence to

132 hydrolyze GTP to GDP ${ }^{12,14}$. ARF proteins are characterized by a unique myristoylated

$133 N$-terminal region, a GTP/GDP-interacting pocket, and two domains mediating binding

134 with regulators and effectors (i.e., switch 1 [SW1] and switch 2 [SW2] regions) that undergo a GTP/GDP-dependent structural rearrangement, allowing the GTPase to interact with effectors in its GTP-bound state ${ }^{15-17}$. Similarly to other members of the RAS superfamily, release of GDP is stimulated by specific guanine nucleotide exchange factors (ARFGEFs), which indirectly favor binding to GTP ${ }^{12,18,19}$. As a consequence of the conformational change promoted by GTP, the $N$-terminal myristoylated region is exposed, allowing anchoring of the GTPase to the cytoplasmic leaflet of membranes of different organelles, including cis and trans-Golgi, plasma membrane (PM) and endosomes, where these proteins exert their function ${ }^{12,14,17,20,21}$.

143 The intrinsic slow GTPase activity of ARFs is substantially accelerated by specific 144 GTPase-activating proteins (ARFGAPs), which result in protein inactivation and release from membranes ${ }^{12,21-23}$.

146 To exert their function, ARF proteins interact with a number of effectors, most of which 147 are coat proteins and adaptors ${ }^{1,12}$; they can also recruit non-coat GA-specific proteins 148 to membranes (e.g., golgin-160 and GCC88) ${ }^{24}$, which are important for GA structure 149 homeostasis ${ }^{25,26}$. Evidence shows that ARF proteins actually contribute to the control 150 of GA and organelles structural organization and function ${ }^{13,27,28}$, and GA dynamics 
Dominantly acting variants in ARF3 have disruptive consequences on Golgi integrity and cause microcephaly recapitulated in zebrafish

Fasano, Muto, Radio, et al.

151 during cell division and cytokinesis in precursor cells ${ }^{29-31}$. By controlling GA structure,

152 function, cargo sorting and ER-GA targeted trafficking, ARFs actively participate to the

153 fine regulation of key events during embryogenesis (i.e., cell polarity establishment and 154 migration in early gastrulation, neuronal maturation and tissue morphogenesis) ${ }^{32}$. 155 Indeed, hyperactive arf1 function in zebrafish results in shortening of the anterior156 posterior (AP) axis, a likely consequence of an altered planar cell polarity (PCP)157 dependent cell movements, which was also shown in a fly wing morphogenesis 158 model $^{33}$. Nevertheless, the underlying mechanisms by which the activity of several 159 ARF proteins on GA organization and trafficking contributes to developmental processes are yet not fully understood.

161 Notwithstanding the pivotal roles of ARF proteins in development, mutations in $A R F$ genes have only recently been linked to human disease, with activating missense 163 variants of ARF1 (MIM: 103180) causing a rare dominant neurodevelopmental disorder 164 (NDD) resulting from defective neuronal migration (MIM: 618185) ${ }^{34}$. Here, we report de novo missense variants in ARF3 underlying a disorder affecting neurodevelopment and causing neurodegeneration in five patients. In silico and in vitro analyses strengthened by in vivo morphometric and live cell behavioral analyses in fish embryos expressing ARF3 mutants provide evidence of a variable impact of mutations on 169 protein function and disruptive consequences on GA integrity, brain and body axes development and PCP-dependent gastrulation processes. These findings demonstrate the relevance of ARF3 function on organelle homeostasis and development. 
Dominantly acting variants in ARF3 have disruptive consequences on Golgi integrity and cause microcephaly recapitulated in zebrafish

Fasano, Muto, Radio, et al.

\section{Results}

\section{ARF3 mutations cause a developmental disorder characterized by microcephaly}

\section{and cortical atrophy}

In the frame of a research program dedicated to subjects affected by unclassified diseases, trio-based exome sequencing allowed us to identify a previously unreported de novo ARF3 variant, c.379A>G (p.Lys127Glu; NM_001659.2), as the putative disease-causing event in a girl (Subject 1) with severe syndromic NDD characterized by growth restriction, microcephaly, progressive diffuse cortical atrophy and other brain anomalies at MRI (i.e., lateral ventricular enlargement, progressive pontocerebellar hypoplasia with major involvement of the cerebellar vermis and hypoplasia of corpus callosum), seizures, inguinal hernia, congenital heart defects (CHD) and skeletal involvement (i.e., 11 rib pairs and scoliosis) (Table 1, Supplementary Figure 1, Supplementary clinical reports). WES data analysis excluded presence of other relevant variants compatible with known Mendelian disorders based on their expected inheritance model and associated clinical presentation, and high-resolution SNP array analysis excluded occurrence of genomic rearrangements. The missense change, which had not previously been reported in population databases, affected an invariantly conserved residue among orthologs, paralogs and other structurally related GTPases of the RAS family (Supplementary Figure 2a).

Through networking and GeneMatcher ${ }^{35}$, we identified four additional subjects with de novo ARF3 missense variants, which had not been reported in EXAC/gnomAD and involved amino acid residues located in regions highly constrained for variation (Supplementary Table 1, Supplementary Figure 2b). No additional candidate variants in clinically associated genes were identified in any patients (WES statistics and data 
Dominantly acting variants in ARF3 have disruptive consequences on Golgi integrity and cause microcephaly recapitulated in zebrafish

Fasano, Muto, Radio, et al.

196

output are reported in Supplementary Tables 2-6). All affected residues were conserved among ARF3 orthologs and paralogs and three of them were also conserved among other RAS GTPases (Supplementary Figure 2a). The identified missense variants affected residues whose corresponding positions in other GTPases of the RAS superfamily had previously been associated with human disease (Supplementary Table 7). The same Lys-to-Glu substitution at codon 127 in Subject 1 was recently reported to affect the corresponding residue in ARF1 in a patient with DD, microcephaly, periventricular heterotopia, progressive cerebral atrophy and epilepsy ${ }^{34}$. Of note, this amino acid is homologous to Lys ${ }^{117}$ in HRAS (MIM: 190020), and an activating missense variant of this residue in HRAS was reported in Costello syndrome (CS [MIM: 218040]) $)^{36}$. Pro ${ }^{47}$ (mutated in Subject 3) is homologous to Pro ${ }^{34}$ in HRAS, KRAS and NRAS (Supplementary Figure 2a). The same Pro-to-Ser change have previously been reported as a somatic event in HRAS in vascular tumors ${ }^{37}$, and changes affecting Pro $^{34}$ in KRAS, HRAS and NRAS have been described in RASopathies ${ }^{38-40}$ ( ClinVar). Moreover, a missense change affecting the adjacent residue in ARF1 (p.Thr48lle) was observed in a patient with clinical features overlapping with the present series ${ }^{40}$. In HRAS and KRAS, mutations affecting $\mathrm{Thr}^{58}$, which is adjacent to the aspartic acid residue homologous to Asp ${ }^{67}$ in ARF3 (mutated in Subject 2), have causally been linked to RASopathies ${ }^{38,41,42}$. Finally, amino acid substitutions at these residues in ARF3, its paralogs and members of the RAS subfamily have been observed as somatic events in malignancies, providing further evidence of their functional and clinical relevance (Supplementary Table 7).

Affected subjects showed variable degree of DD/ID (Table 1, Supplementary clinical reports). No characteristic craniofacial gestalt was noted, with only minor craniofacial 
Dominantly acting variants in ARF3 have disruptive consequences on Golgi integrity and cause microcephaly recapitulated in zebrafish

Fasano, Muto, Radio, et al.

features reported, which were mainly related to microcephaly. Likewise Subject 1 , Subject 2 showed microcephaly, profound DD, progressive diffuse cortical atrophy with diminished hemispheric white matter, with a thin corpus callosum, progressive pontocerebellar hypoplasia, hypotonia, microsomia, and skeletal defects. A comparable condition was also observed in Subjects 3 and 4, who manifested hypotonia, variable DD/ID, microcephaly, and various MRI anomalies (Table 1). In Subject 5, global DD and cognitive deficits were associated with hypoplasia of the corpus callosum and mild white matter involvement in periventricular and supraventricular areas, with normal head circumference (Supplementary Figure 1). Early-onset seizures had been reported in Subjects 1 and 3.

\section{Disease-associated $A R F 3$ variants variably affect protein stability and function}

The identified disease-associated variants affected residues spotted throughout the coding sequence with exception of the $C$-terminus region (Figure 1a). First, we investigated the functional consequences of each amino acid substitution by using a three-dimensional structure of the GTPase recently solved by X-ray diffraction ${ }^{43}$. We noted that all residues cluster within or close to the GTP/GDP binding pocket (Figure 1b). Specifically, Lys ${ }^{127}$ is one of the four residues of the NKXD motif directly mediating binding to GTP/GDP by binding to the ribose ring ${ }^{16}$, and substitution of the positively charged residue with a negatively charge glutamate was predicted to affect proper nucleotide binding (Figure 1c). Similarly, Thr $^{32}$ contributes to stabilize the GTP/GDP binding via direct hydrogen bonding with one oxygen atom of the $\alpha$ phosphate (Figure 1c). While conservative, the Thr to Asn substitution was predicted to result in a steric hindrance. Asp ${ }^{93}$ does not directly contact GTP, even though it participates to the overall general structure of the nucleotide binding pocket by a direct hydrogen bond 
Dominantly acting variants in ARF3 have disruptive consequences on Golgi integrity and cause microcephaly recapitulated in zebrafish

Fasano, Muto, Radio, et al.

244 with the lateral chain of Lys ${ }^{127}$ (Figure 1d). Since the high GTP:GDP ratio within cells,

245 these three changes were anticipated to favor an active, GTP-bound state of the

246 GTPase, bypassing the requirement for a GEF, as previously reported for pathogenic 247 mutations affecting RAS proteins ${ }^{44,45}$. On the other hand, Pro ${ }^{47}$ and $\mathrm{Asp}^{67}$ were 248 predicted to affect ARF3 GTPase activity. Specifically, Pro ${ }^{47}$ is located within the switch 1 region, which plays a key role in the catalytic activity of the GTPase and the conformational rearrangement mediating binding to effectors ${ }^{12,16}$. Substitution of this non-polar residue with a polar serine is expected to strongly perturb the functional behavior of the protein. Similarly, Asp ${ }^{67}$ participates in the coordination of the $\mathrm{Mg}^{2+}$ ion through direct hydrogen bonds with a water molecule ${ }^{15}$ (Figure 1c), and contributes to the regulation of GDP/GTP binding upon the "inter-switch toggle" mechanism ${ }^{46}$; its substitution with valine was predicted to considerably perturb the GTP/GDP binding switch $^{17}$ and the overall organization of the nucleotide binding pocket. No obvious consequence was hypothesized for the substitution involving Leu ${ }^{12}$ (found in cis with p.Asp67Val in Subject 2) which resides in the flexible $N$-terminal domain of the GTPase. Of note, while $\mathrm{Thr}^{32}$, Asp ${ }^{93}$ and Lys ${ }^{127}$ map regions of the GTPase not directly involved in intermolecular contacts, $\mathrm{Pro}^{47}$ and $\mathrm{Asp}^{67}$ lie in regions close to the surface of the GTPase interacting with effectors/regulators ${ }^{47}$, which does not rule out the possibility of a more complex functional behavior of the p.Pro47Ser and p.Asp67Val changes. To explore the structural and functional consequences of these two amino acid substitutions, we built a model of ARF3 interacting with the cytosolic coat protein complex (COP) formed by $\mathrm{y}-\mathrm{COP}(\mathrm{COPG} 1)$ and $\zeta-\mathrm{COP}$ (COPZ1) starting from an available GTP-bound ARF1:COPG1-COPZ1 complex (PDB: 3TJZ) as template ${ }^{48}$. The model for the wild-type (WT) ARF3 protein was validated by a 500-ns molecular dynamics (MD) simulation, documenting conservation of all known interactions with 
Dominantly acting variants in ARF3 have disruptive consequences on Golgi integrity and cause microcephaly recapitulated in zebrafish

Fasano, Muto, Radio, et al.

GTP and Mg2+ (Figure 1d,e; Supplementary Table 8). The ARF3:COPG1 interface is stabilized by an intermolecular hydrogen bonding network involving $\mathrm{Arg}^{19}$, $\mathrm{Thr}^{48}$ and

9). We assessed the structural perturbations due to the introduced p.Pro47Ser and p.Asp67Val changes using the same time-frame. A minor impact on the ARF3 surface interacting with COPG1 was evident in the simulation when introducing the Asp67Val substitution (Figure 1f;

Supplementary Table 9). As predicted by the structural inspection, this change resulted instead in a significant rearrangement of the nucleotide binding pocket with a reduction of the interactions of $\operatorname{Lys}^{127}$ and $\mathrm{Thr}^{45}$ with GTP (Supplementary Table 8). The Pro-toSer substitution at codon 47 did not significantly affect ARF3 binding to GTP (Supplementary Table 8), while a dramatic perturbation of the intermolecular binding network with COPG1 due to a substantial rearrangement of the Switch 1 region was observed (Figure 1g; Supplementary Table 9). Consistently, essential dynamics analysis documented a major effect of Pro47Ser in terms of global fluctuations and long range correlated movements, compared to the other simulations (Supplementary

Figure 3). Overall, the structural analyses predicted that all variants but p.Lys 12 Val affect GTP/GDP binding and/or the GTPase activity of the protein, promoting an overall stabilization of ARF3 in its catalytically active conformation. A more articulated impact on conformational rearrangements mediating binding to effectors was suggested for p.Pro47Ser.

To experimentally validate the predicted consequences of the identified variants on ARF3 function, we investigated the biochemical behavior of a subset of mutants (i.e., $A R F 3^{P 47 S}, A R F 3^{L 12 V ; D 67 V}$ and $\left.A R F 3^{K 127 E}\right)$ in vitro. First, we examined the protein levels of the selected ARF3 mutants in transiently transfected COS-1 cells. Immunoblotting 
Dominantly acting variants in ARF3 have disruptive consequences on Golgi integrity and cause microcephaly recapitulated in zebrafish

Fasano, Muto, Radio, et al.

293

294

295

296

297

298

299

300

301

302

303

304

305

306

307

308

309

analysis documented that ARF $3^{\text {P47S }}$ level was comparable to the WT protein, while we observed a considerably reduced level for both the ARF3 $3^{\mathrm{K} 127 \mathrm{E}}$ and ARF3 ${ }^{\mathrm{L} 12 \mathrm{~V} ; \mathrm{D} 67 \mathrm{~V}}$ mutants (Figure 2a), which was confirmed also in vivo (Supplementary Figure 4). Treatment with the proteasome inhibitor MG132 and the autophagy inhibitor bafilomycin A1 partially restored the levels of both mutants, indicating a reduced stability and accelerated degradation (Figure 2b).

In its active GTP-bound state, ARF3 is able to bind to the Golgi-associated gammaadaptin ear-containing ARF-binding protein 3 (GGA3) to regulate downstream events controlling trans-Golgi function and intracellular trafficking ${ }^{49}$. Thereby, we performed pull-down experiments using the GGA3 protein-binding domain (PBD) on cell lysates to compare the relative amounts of GTP-bound fraction of WT and mutant ARF3 proteins and their ability to transduce signaling through proper binding to effectors. Immunoblot analysis revealed a basal increase of the GTP-bound fraction for all mutants compared to WT ARF3, which was statistically significant for ARF3 ${ }^{\mathrm{K} 127 \mathrm{E}}$ and $A R F 3^{L 12 V ; D 67 V}$ (Figure 2c). These data are consistent with the MD predictions and indicate a stabilized GTP-bound conformation and an overall hyperactive behavior of the microcephaly-associated variants.

Golgi integrity is altered in cells expressing the disease-associated ARF3 mutants

The intracellular localization of ARF proteins is highly dynamic. GTP-bound ARF3 specifically localizes at the trans-Golgi,where it coordinates vesicle budding ${ }^{14,50}$. Based on these considerations and previous observations indicating the relevant role of ARF proteins in maintaining proper organization and function of $\mathrm{GA}^{4,28,51}$, we examined the subcellular distribution of $C$-terminal mCherry-tagged ARF3 mutants and evaluated the 
Dominantly acting variants in ARF3 have disruptive consequences on Golgi integrity and cause microcephaly recapitulated in zebrafish

Fasano, Muto, Radio, et al.

317 morphology of the trans-Golgi compartment using EGFP-tagged galactosidase T 318 (GalT) as a marker ${ }^{52}$, in transfected COS-1 cells and zebrafish embryos (Figure 3a).

319 As expected, live cell imaging by confocal microscopy documented a diffuse localization of WT ARF3 in cells (GDP-bound ARF3) already from 4 hours after transfection, and its co-localization with EGFP-GalT (GTP-bound ARF3), which showed a compact morphology of the intact trans-Golgi (Figure 3b; Supplementary

Video 1). In striking contrast, cells overexpressing ARF3 ${ }^{\mathrm{K} 127 \mathrm{E}}$ were characterized by a dispersed EGFP-GalT signal over time (Figure 3c; Supplementary Video 1), indicating a fragmentation of the trans-Golgi structure. This finding was confirmed by analyzing fixed cells overexpressing $\mathrm{ARF}^{\mathrm{K} 127 \mathrm{E}}$ also 24 hours after transfection by immunofluorescence analysis against golgin 97 , whose staining appeared weak and dispersed compared to what observed in cells overexpressing WT ARF3 (Supplementary Figure 5a,b). Double labeling experiments and quantitative analysis were extended to other ARF3 mutants, which confirmed a variable scattered/absent golgin 97 signal, compared to the perinuclear compact and intense staining documented in cells expressing the WT protein (Supplementary Figure 5c,d).

Fragmented trans-Golgi was observed in all the examined cells expressing ARF $3^{\mathrm{K} 127 \mathrm{E}}$ and $A R F 3^{L 12 V ; D 67 V}$ and in a relevant proportion of cells expressing $A R F 3^{P 47 S}$ (approximately 40\%) (Supplementary Figure 5e). To further validate these findings in vivo, we microinjected WT and mutant mCherry-tagged ARF3 mRNAs in zebrafish embryos at the one-cell stage and assessed protein intracellular localization in superficial cells (envelope layer cells, ELC) of early gastrula (Figure 3a,d,e). Consistent with the in vitro findings, we observed a diffused distribution of WT ARF3 in cells, which partially overlapped with EGFP-GalT staining (Figure 3d). Conversely, ARF3 ${ }^{\mathrm{K} 127 \mathrm{E}}$ distribution in cells appeared markedly restricted, organized in small puncta, and co- 
Dominantly acting variants in ARF3 have disruptive consequences on Golgi integrity and cause microcephaly recapitulated in zebrafish

Fasano, Muto, Radio, et al.

342 localized with EGFP-GalT (Figure 3e), providing evidence of a strict localization at the

343 trans-Golgi and thereby of a stabilized GTP-bound state of the mutant protein.

344 Confirming the in vitro observations, differently from what observed in ELC cells in embryos overexpressing WT mCherry-tagged ARF3, EGFP-GalT staining appeared reduced and more scattered (with an increase of cells showing "puncta" morphology) in embryos injected with $A R F 3^{\mathrm{K} 127 \mathrm{E}} \mathrm{mRNA}$, suggesting trans-Golgi fragmentation

348 (Figure 3e, yellow arrows, Supplementary Figure 6). Overall, these data suggest that 349 the p.Lys127Glu and p.Leu12Val;p.Asp67Val changes force ARF3 into a GTP-locked 350 (active) state, promoting a stable binding of the GTPase to the trans-Golgi membrane that, in turn, affects Golgi integrity, resembling what previously observed for a constitutively active (GTP-bound) ARF1 mutant ${ }^{51}$. To a minor extent, this effect was also documented for the ARF3 ${ }^{\mathrm{P} 47 \mathrm{~S}}$ mutant.

Expression of $A R F 3^{K 127 E}$ and $A R F 3^{L 12 V ; D 67 V}$ in zebrafish embryos causes pleiotropic effects on development and recapitulates the microcephalic trait

Next, we analyzed the impact of the disease-associated missense changes in early zebrafish development. WT and mutant myc-tagged ARF3 mRNAs were microinjected together with the membrane maker GFP-CAAX mRNA at one cell stage

359 (Supplementary Figure 7a). Few hours after injection, embryos in their cleavage period were sorted based on the expression of GFP-CAAX (Supplementary Figure 7b, left), and developmental progression was followed from early time points of gastrulation till 48 hpf embryos (long-pec stage), when morphogenesis is nearly completed and subcompartmentalization of different neural structures can be appreciated ${ }^{53}$. Upon 364 standard rearing conditions, we observed a statistically significant decrease in the 365 survival rate of embryos expressing each mutant at $24 \mathrm{hpf}$ and $48 \mathrm{hpf}$, compared to 
Dominantly acting variants in ARF3 have disruptive consequences on Golgi integrity and cause microcephaly recapitulated in zebrafish

Fasano, Muto, Radio, et al.

those expressing WT ARF3 and their not-injected siblings (Supplementary Figure 7b, right), with the strongest effect observed for ARF3 ${ }^{\mathrm{K} 127 \mathrm{E}}$. Development appeared perturbed both at the level of the head and trunk (Supplementary Figure 7c-e). At 24 hpf, the severe phenotype (presence of microcephaly, microphthalmia, defective body elongation and severe lateral bending) was highly prevalent in embryos expressing $\mathrm{ARF}^{\mathrm{K} 127 \mathrm{E}}(45.4 \%)$ (Supplementary Figure $\left.7 c^{\prime}, \mathrm{e}\right)$, and occurred in a small proportion of embryos expressing the ARF3 $3^{\mathrm{L} 12 \mathrm{~V} ; \mathrm{D} 67 \mathrm{~V}}$ mutant (3.8\%) (Supplementary Figure 7c",e). While a negligible fraction of embryos expressing the WT protein $(4.3 \%)$ was mildly affected, this proportion was significantly higher in fish expressing each mutant $(74 \%$, ARF3 $^{\mathrm{K} 127 \mathrm{E}} ; 27 \%, \mathrm{ARF}^{\mathrm{L} 12 \mathrm{~V} ; \mathrm{D} 67 \mathrm{~V}}$ ) (Supplementary Figure 7e). A similar incidence was observed even halving the concentration of injected ARF mutant mRNAs (Supplementary Figure 8a). The phenotype worsened with time for both mutants, with the most severe cases reaching $57 \%$ for $\mathrm{ARF}^{\mathrm{K} 127 \mathrm{E}}$ and $17 \%$ for $\mathrm{ARF} 3^{\mathrm{L} 12 \mathrm{~V} ; \mathrm{D} 67 \mathrm{~V}}$ by the time of hatching (48 hpf) (Supplementary Figure 8b, c).

When analyzed at $48 \mathrm{hpf}$, microcephaly was evident in zebrafish expressing mutant ARF3 alleles (Figure 4a-c'). Indeed, embryos injected with $A R F 3^{\mathrm{K} 127 \mathrm{E}}$ and $A R F 3^{L 12 V ; D 67 V}$ mRNAs showed a significant reduction of the head area compared to animals expressing WT ARF3 and not injected controls, with the most severe cases lacking the frontal part of the brain and eyes (Figure 4a,c), as observed already at 24 hpf (Supplementary Figure $\left.7 \mathrm{~d}, \mathrm{~d}^{\prime}\right)$. To validate the significance of the observed head reduction, next we injected $A R F 3^{W T}$ and $A R F 3^{K 127 E}$ mRNAs in the transgenic NBT:dsRed line, labeling differentiated neurons (Figure 4b). Volumetric reconstructions and measurements from live confocal z-stack acquisitions at $48 \mathrm{hpf}$ confirmed a significant reduction of the brain volume (Figure $4 \mathrm{~b}, \mathrm{c}^{\prime}$ ). Additional 
Dominantly acting variants in ARF3 have disruptive consequences on Golgi integrity and cause microcephaly recapitulated in zebrafish

Fasano, Muto, Radio, et al.

390

391

392

393

394

395

396

397

398

399

400

401

402

403

404

405

406

407

408

409

410

411

412

413

volumetric measurements obtained from fixed specimens at $48 \mathrm{hpf}$ by labeling mature brain structures using anti-acetylated alpha-tubulin and anti-HuC/Elav antibodies (Supplementary Figure 9) further confirmed the deleterious effect of the ARF3 ${ }^{\mathrm{K} 127 \mathrm{E}}$ allele on brain development.

Next, to investigate the developmental processes implicated in the body curvature defects observed in the embryos expressing the ARF3 mutants, we analyzed the morphology and development of the notochord, which is crucial to support the body elongation along the anterior to posterior axis (AP) and for subsequent spine formation ${ }^{54}$. At $30-35 \mathrm{hpf}$, when notochord morphogenesis is almost concluded, confocal imaging analysis documented the occurrence of multiple notochord curvatures per embryo in animals expressing each of the two mutant mRNAs, which were distributed throughout the AP axis and were characterized by bending of variable degrees (Figure 4d-f). In agreement with the overall severity of the observed phenotype, ARF3 $3^{\mathrm{K} 127 \mathrm{E}}$ showed a significant higher number of notochord curvatures. On the other hand, quantification of the degree of bending $\left(180^{\circ}\right.$ : normal; $179^{\circ} \geq$ angle $\geq 110^{\circ}$ : mild; angle $\leq 109^{\circ}$ : severe) documented equivalent involvement in embryos expressing the two ARF mutants. No gross phenotype related to either brain or body axis morphology and size was observed in embryos expressing WT ARF3 and in noninjected control siblings. Overall, these data indicate that the severe reduction of the head in animals expressing mutant ARF3 proteins is caused by an impaired brain development, recapitulating patients' microcephaly and that the observed embryo curvatures are due to a perturbed notochord development.

ARF3 $^{\mathrm{K} 127 \mathrm{E}}$ and ARF3 ${ }^{\mathrm{L} 12 \mathrm{~V} ; \mathrm{D} 67 \mathrm{~V}}$ alter PCP-dependent processes in early zebrafish development 
Dominantly acting variants in ARF3 have disruptive consequences on Golgi integrity and cause microcephaly recapitulated in zebrafish

Fasano, Muto, Radio, et al.

414 We investigated further the perturbed head-trunk morphogenesis by tracing back axes

415 establishment in embryos expressing WT and mutant ARF3 proteins. First, we 416 examined patterning and morphogenesis in animals in their segmentation period (15 $417 \mathrm{hpf}, 13-14$ somites). During this period, forebrain, midbrain and hindbrain are 418 discernible, the embryo $\mathrm{AP}$ and $\mathrm{ML}$ axes are already established, and the embryo 419 actively elongates with the tail bud protruding together with somites formation. mRNA 420 levels of Krox20, which is expressed in anterior cephalic domain (hindbrain 421 rhombomeres) and $M y o D$, expressed at the level of the trunk paraxial mesoderm 422 (developing somites), was assessed in whole-mount embryos by In situ hybridization 423 (ISH) (Figure 5a). While proper patterning of cephalic hindbrain region and paraxial mesoderm was in place, we observed a significant perturbation of the AP and ML axes 425 (Figure 5b-f), which are defects typically linked to alteration of planar cell polarity (PCP)-controlled processes ${ }^{55,56}$ and consistent with previous observations reported in fish embryos expressing mutant ARF1 proteins ${ }^{33}$. In detail, compared to their control siblings, mutant fish showed a clear shortening of the AP length and an expansion of the paraxial tissue in the ML axis (Figure 5b). Morphometric analysis of the Krox20 and MyoD mRNA spatial expression profiles revealed a significant reduction of the AP 431 length in the mutant embryos (Figure $5 b, c$ ) and a significant enlargement of the ML axis was evident in embryos expressing ARF3 ${ }^{\mathrm{K} 127 \mathrm{E}}$ (Figure 5b,d,e). Consistently, the number of somites in mutant embryos was also reduced (Supplementary Figure 10).

434 For both AP and ML axes defects, severely affected embryos were more prevalent 435 among those expressing ARF3 ${ }^{\mathrm{K} 127 \mathrm{E}}$ (Figure 5f).

Shortening and alteration of the body axes normally depend upon defective 437 gastrulation occurring early during embryogenesis. At this developmental stage, cells 
Dominantly acting variants in ARF3 have disruptive consequences on Golgi integrity and cause microcephaly recapitulated in zebrafish

Fasano, Muto, Radio, et al.

undergo a complex series of movements converging towards the dorsal midline and extending anteriorly that are controlled by trafficking and asymmetrical positioning of PCP components ${ }^{55-57}$. To clarify the mechanism by which overexpression of activating ARF3 proteins causes severe axes perturbation in zebrafish development, first we investigated possible extension defects by measuring animal elongation in embryos at around $13 \mathrm{hpf}$ (Figure 6a). At this stage, as a result of correctly orchestrated gastrulation movements, brain thickenings and tail bud are visible at the very anterior and posterior end of the embryo, respectively ${ }^{53}$. Compared to embryos injected with WT ARF3 mRNA, a statistically significant increase of the angle between the developed cephalic and caudal structures was observed in embryos expressing $\mathrm{ARF}^{\mathrm{K} 127 \mathrm{E}}$ (Figure $6 \mathrm{~b}, \mathrm{c}$ ), which is indicative of delayed extension cell movements. Of note, embryos expressing the two mutants showed a statistically significant difference in the extent of early body elongation, which was in line with the difference observed during later segmentation stage (15 hpf) and at $24 \mathrm{hpf}$. In addition, live confocal imaging performed during gastrulation in individual $A R F 3^{W T}$ or $A R F 3^{K 127 E}-m C h e r r y$ expressing embryos documented delayed epiboly in animals expressing the ARF3 mutant (Figure 6d,e). We also observed defective gastrulation movements in embryos expressing ARF $3^{\mathrm{K} 127 \mathrm{E}}$ during the entire time-lapse, resulting in defective head and tail bud formation by the end of the acquisition.

These findings suggest that compromised convergent extension movements resulting from $A R F 3^{K 127 E}$ overexpression during gastrulation lead to an impairment of PCPdependent body axes establishment. Cells expressing the mutant appeared mostly round in shape, with a reduced number of protrusions, with respect to cells expressing the WT protein (close-up in Figure 6d,e and Figure 6f), suggesting the possibility of an 
Dominantly acting variants in ARF3 have disruptive consequences on Golgi integrity and cause microcephaly recapitulated in zebrafish

Fasano, Muto, Radio, et al.

462

altered polarity establishment and cytoskeletal organization as a molecular event contributing to the defective cell motility.

\section{Discussion}

Here we identify de novo activating missense variants of $A R F 3$ as the molecular event underlying a neurodevelopmental condition characterized by microcephaly, progressive cerebral atrophy, and DD/ID, with short stature and skeletal abnormalities variably occurring as associated features. The phenotype of this disorder is reminiscent of the condition caused by activating mutations in $A R F 1^{34}$. Consistently, we show that disease-causing ARF3 variants are variably activating, stabilizing the protein in its GTP-bound conformation. Importantly, we also document that these dominantly acting amino acid substitutions affect Golgi integrity, and perturb brain formation and embryonic axes development in a vertebrate model, tracing back their first assessable effect to defective PCP-mediated cell motility in early precursor cells.

The small GTPases of the ARF family are highly conserved across eukaryotes, occupy different subcellular compartments involved in ER-GA network, and have both redundant and distinct functions ${ }^{11}$. These proteins control key molecular and cellular processes, including targeted intracellular trafficking of signaling proteins, cell migration and division, lipid metabolism, and signaling, which are important during animal development ${ }^{1,32,33}$. The intracellular localization and activity of ARF3 at the trans-Golgi, where it promotes recruitment of coat complexes and vesicle formation, is tightly regulated via a conformational switch controlled by reversible GDP-to-GTP binding $^{12,16}$. By structural inspection, we demonstrated that disease-causing ARF3 mutations affect conserved residues involved in GDP/GTP binding/exchange, and pull- 
Dominantly acting variants in ARF3 have disruptive consequences on Golgi integrity and cause microcephaly recapitulated in zebrafish

Fasano, Muto, Radio, et al.

down assays experimentally confirmed that two of the three tested mutants stabilize the GTP-bound conformation of the protein, similarly to what has previously been observed for other small monomeric GTPases of the RAS superfamily (e.g., ARF1, HRAS, KRAS, NRAS, MRAS, RRAS, RRAS2) implicated in human disease ${ }^{34,58-}$ ${ }^{63}$ (COSMIC database ). The functional relevance of this perturbed equilibrium between the inactive and active state of the GTPase was further evidenced in vivo by documenting a strict localization of the ARF $3^{\mathrm{K} 127 \mathrm{E}}$ protein at the trans-Golgi in zebrafish embryos. It should be mentioned that two mutations (p.Pro47Ser and p.Asp67Val) affected a conserved hydrophobic region involved in effector binding ${ }^{17,64,65}$, with molecular dynamics simulations suggesting a major perturbation exerted by the p.Pro47Ser substitution on ARF3 binding to effectors. These considerations stimulate future studies aimed to demonstrate whether effector binding in these mutants is qualitatively and/or quantitatively altered.

ARF1 and ARF3 are known to play a key role in maintaining organelle integrity $27,28,66$. Xiang et al. (2007) ${ }^{51}$ showed that physiological GA fragmentation in mitotic cells is principally mediated by the active form of ARF1 (GTP-bound), which triggers the continued budding of COPI vesicles. Similarly, while depletion of ARF1 was not reported to affect GA function ${ }^{11}$, disruption of the GA network was observed in cells expressing a constitutively active ARF1 mutant ${ }^{51}$, causing GA swelling with sustained vesiculation ${ }^{13}$. Consistent with the role of these proteins in maintaining organelles structural organization, live imaging in cells expressing the ARF ${ }^{\mathrm{K} 127 \mathrm{E}}$ protein both in vitro and during early zebrafish gastrulation documented a strong and rapid effect on GA integrity, indicating that a shift of the equilibrium towards the GTP-bound form of ARF3 dramatically affect trans-Golgi morphology and embryo development. The 
Dominantly acting variants in ARF3 have disruptive consequences on Golgi integrity and cause microcephaly recapitulated in zebrafish

Fasano, Muto, Radio, et al.

510 molecular mechanism by which aberrant ARF3 function causes GA fragmentation and

511 whether this impacts vesicle biogenesis and trafficking remains to be determined.

512 The finding of fragmented GA in cells and embryos expressing the disease-associated

513 ARF3 mutants assigns this disorder to the recently defined family of "Golgipathies", a

514 group of neurodevelopmental disorders clinically characterized by a wide spectrum of

515 central nervous system abnormalities ${ }^{8,9}$. Similar to what was observed in the present

516 cohort, patients with activating ARF1 pathogenic variants show DD/ID, microsomia,

517 microcephaly and brain abnormalities, including delayed myelination, cortical and

518 cerebellar atrophy, and seizures as major features. Different from what observed in the

519 present series, periventricular heterotopia is a recurrent feature in patients with activating ARF1 variants. A related but distinct neurodevelopmental disorder, periventricular heterotopia with microcephaly (ARPHM, MIM: 608097), has been linked

522 to biallelic inactivating variants of $A R F G E F 2$. This recessive disorder is characterized

523 by severe DD/ID, epilepsy, brain atrophy and delayed myelination associated with thin corpus callosum ${ }^{67}$. Together with previous findings, our work documents the critical role of GA for diverse neurodevelopmental processes as well as for neuronal function.

Our in vivo results deepen the understanding on the impact of dominant $A R F 3$ mutations on development. Post-Golgi cell trafficking and correct sorting of polarity components are required for asymmetric cell division and migration in the vertebrate brain, underlying neurogenesis, axon arborization and sustained synaptogenesis ${ }^{32,68}$.

530 In line with this evidence and the features documented in patients with mutated ARF3 531 alleles, transient overexpression of the $\mathrm{ARF} 3^{\mathrm{K} 127 \mathrm{E}}$ protein coupled to volumetric 532 imaging reconstruction and live cell analysis in zebrafish embryos reveled a clear 533 microcephalic trait and occurrence of defects linked to altered PCP signaling during 
Dominantly acting variants in ARF3 have disruptive consequences on Golgi integrity and cause microcephaly recapitulated in zebrafish

Fasano, Muto, Radio, et al.

early gastrulation.

535 Convergence and extension movements, which require a fine tuning of cell polarity

536 mechanisms and are needed to shape the AP and ML axes and proper head and trunk 537 domains ${ }^{56}$, were evidently affected in animals expressing the ARF3 mutants. 538 Biosynthetic trafficking and a correct function of ER and GA is essential during animal development for regulating morphogens' distribution ${ }^{32,33,69}$, which include components of the PCP pathway and cadherins required for cell polarity establishment and

541 migration, as shown in vitro ${ }^{70}$, nematodes ${ }^{71}$ and zebrafish ${ }^{72}$. Consistent with our

542 findings, fish expressing the hyperactive microcephaly-associated ARF1 mutant show

543 PCP-related axial defects ${ }^{33}$, and fish mutants for ARF-interacting COPI/COPII coat 544 components exhibit skeletal and notochord abnormalities with GA disruption ${ }^{73,74}$. Of note, the impaired function of ARFGEF2 underlying the microcephalic traits observed

546 in patients with ARPHM, has been linked to proliferative and migratory defects due to 547 trans-Golgi to membrane trafficking of E-cadherins and beta-catenin ${ }^{75}$. Altogether, this body of evidence points to an important role of ARF-mediated trafficking of signaling components during brain and body plan development deserving further investigation.

In conclusion, our findings highlight a role of ARF3 in the maintenance of trans-Golgi integrity, and document an obligate dependence of early developmental processes and

552 brain morphogenesis on proper function of this GTPase, identifying ARF3 as a novel 553 gene implicated, when mutated, in a neurodevelopmental disorder belonging to the emerging class of "Golgipathies" ARF3-driven disruption of GA integrity impacts developmental signaling homeostasis throughout embryogenesis, and thereby the cellular processes underlying brain 557 formation. 
Dominantly acting variants in ARF3 have disruptive consequences on Golgi integrity and cause microcephaly recapitulated in zebrafish

Fasano, Muto, Radio, et al.

\section{Methods}

\section{Subjects}

560 The study has been approved by the local Institutional Ethical Committee of the

561 Ospedale Pediatrico Bambino Gesù IRCCS (OPBG), Rome (1702_OPBG_2018).

562 Subject 1 was analyzed in the frame of a research project dedicated to undiagnosed 563 disorders (Undiagnosed Patients Program, OPBG), while the other subjects were

564 referred for diagnostic genetic testing. Clinical data and DNA samples were collected,

565 stored and used following procedures in accordance with the ethical standards of the

566 declaration of Helsinki protocols, and after signed consents from the participating 567 families. Permission was obtained to publish photographs of Subjects 1 and 4.

\section{Exome sequencing analysis}

569 In all families, whole-exome sequencing (WES) was performed using DNA samples

570 obtained from leukocytes and a trio-based strategy was used. Target enrichment kits, 571 sequencing platforms, data analysis, and WES statistics are reported in 572 Supplementary Table 2-6 and in the Supplementary Methods. WES data processing, 573 read alignment to the GRCh37/hg19 version of genome assembly, and variant filtering 574 and prioritization by allele frequency, predicted functional impact, and inheritance 575 models were performed as previously reported ${ }^{76-80}$. WES data output is summarized 576 in Supplementary Table 2-6. Cloning of the genomic portion encompassing the 577 c.34C>G and c.200A>T missense substitutions (p.Leu12Val and p.Asp67Val; Subject 578 3) was used to confirm that both variants were on the same allele. Variant validation 579 and segregation were assessed by Sanger sequencing in all the subjects included in 580 the study. 
Dominantly acting variants in ARF3 have disruptive consequences on Golgi integrity and cause microcephaly recapitulated in zebrafish

Fasano, Muto, Radio, et al.

581

582

583

584

585

586

587

588

589

590

591

592

593

594

595

596

597

598

599

600

601

602

603

\section{Structural analysis and molecular dynamics simulations}

The structural impact of the disease-associated missense changes was assessed using the available three-dimensional structures of human ARF3 complexed with GTP and V. vulnificus multifunctional-autoprocessing repeats-in-toxin (MARTX) (PDB $6 \mathrm{ii6})^{43}$.The structure was visualized using the VMD visualization software ${ }^{81}$.

A model of GTP-bound ARF3 interacting with the cytosolic coat protein complex subunits $\mathrm{Y}$-COP (COPG1) and $\zeta$-COP (COPZ1) was built using the SWISS-MODEL automated protein structure homology modelling server (http://swissmodel.expasy.org) ${ }^{82}$ using the $2.90 \AA$ resolution X-ray structure (PDB $3 \mathrm{TJZ})^{48}$. Alignment of template and model amino acid sequences is reported in Supplementary Figure 11. The p.Asp67Val and p.Pro47Ser mutations were introduced using the UCSF Chimera package ${ }^{83}$. The side-chain orientations were obtained with the Dunbrack backbone-dependent rotamer library ${ }^{84}$, choosing the best rotamer with minimal/no steric clashes with neighboring residues. Following protonation of titratable amino acids at $\mathrm{pH}=7$, proteins were added in cubic boxes and solvated in water. Counter-ions were added to neutralize the charges of the system with the genion GROMACS tool ${ }^{85}$. After energy minimizations, the systems were slowly relaxed for 5 $\mathrm{ns}$ by applying positional restraints of $1000 \mathrm{~kJ} \mathrm{~mol}^{-1} \mathrm{~nm}^{-2}$ to atoms. Unrestrained simulations were carried out for a length of 500 ns with a time step of 2 fs using GROMACS 2020.2. The CHARMM36 all-atom force field ${ }^{86}$ was used for the protein description and water molecules were described by TIP3P model ${ }^{104}$. V-rescale temperature coupling was employed to keep the temperature constant at $300 \mathrm{~K}^{87}$. The Particle-Mesh Ewald method was used for the treatment of the long-range electrostatic 
Dominantly acting variants in ARF3 have disruptive consequences on Golgi integrity and cause microcephaly recapitulated in zebrafish

Fasano, Muto, Radio, et al.

604 interactions ${ }^{88}$. The first 5 ns portion of the trajectory was excluded from the analysis.

605 All analyses were performed using GROMACS utilities.

606 Expression constructs and in vitro mRNA synthesis

607 The full-length coding sequence of WT human ARF3 (NM_001659.3) was obtained by 608 PCR and cloned into the pcDNA3.1/myc-6His eukaryotic expression vector (Life

609 Technologies). The disease-associated substitutions were introduced by site-directed 610 mutagenesis (QuikChange II Site-Directed Mutagenesis Kit, Agilent Technologies). For 611 zebrafish expression experiments, the myc-tagged ( $C$-terminus) WT and mutant $A R F 3$ 612 sequences were subcloned into the pCS-Dest vector (plasmid 22423, Addgene) ${ }^{89}$ via 613 LRII clonase-mediated recombination (ThermoFisher). pCS-Dest-mKOFP2-CAAX and 614 pCS-Dest-EGFP-GalT were generated by subcloning the Addgene plasmids $75155^{90}$ and $11929^{91}$, respectively. Plasmids were digested and linearized with Kpnl (NEB New

616 England Biolabs), and mRNA was produced using mMessage mMachine SP6 617 transcription kit and poly(A) tailing kit (Thermo Fisher). The GFP-CAAX expressing 618 plasmid was a gift from Dr. M. Handberg Thorsager. For live confocal experiments, 619 pcDNA3/hARF3(WT)-mCherry (plasmid 79420, Addgene) ${ }^{92}$ was used and subcloned 620 into zebrafish pCS-Dest vector. All cloned sequences were confirmed by bidirectional 621 DNA sequencing.

\section{COS-1 cell culture and transient transfection assays}

623 COS-1 cells were cultured in Dulbecco's modified Eagle's medium supplemented with $10 \%$ heat-inactivated fetal bovine serum (GIBCO), $1 \mathrm{x}$ sodium pyruvate and $1 \mathrm{x}$ penicillin-streptomycin, at $37{ }^{\circ} \mathrm{C}$ with $5 \% \mathrm{CO}_{2}$. Subconfluent cells were transfected with myc- or mCherry-tagged WT and mutant ARF3 expressing vectors using FuGENE 6

627 (Promega), according to the manufacturer's instructions. 
Dominantly acting variants in ARF3 have disruptive consequences on Golgi integrity and cause microcephaly recapitulated in zebrafish

Fasano, Muto, Radio, et al.

628

629

630

631

632

633

634

635

636

637

638

639

640

641

642

643

644

645

646

647

648

649

650

651

\section{ARF3-myc immunoblotting}

Transfected COS-1 cells were lysed in radio-immunoprecipitation assay (RIPA) buffer, pH 8.0, containing phosphatase and protease inhibitors (Sigma-Aldrich). Lysates were kept on ice for $30 \mathrm{~min}$ and centrifuged at $16,000 \mathrm{~g}$ for $20 \mathrm{~min}$ at $4{ }^{\circ} \mathrm{C}$. Samples containing an equal amount of total proteins $(15 \mu \mathrm{g})$ were resolved by $12 \%$ sodium dodecyl sulfate (SDS)-polyacrylamide gel (Biorad). Proteins were transferred to nitrocellulose membrane using a dry transfer system (Biorad), and blots were blocked with $5 \%$ non-fat milk powder (Biorad) in Phosphate-buffered saline (PBS) containing $0.1 \%$ Tween-20 for $1 \mathrm{~h}$ at $4{ }^{\circ} \mathrm{C}$ and incubated with mouse monoclonal anti-myc (1:1000, Cell Signaling), mouse monoclonal anti- $\beta$-tubulin (1:1000, Sigma-Aldrich) and antimouse HRP-conjugated secondary antibody (1:3000; Sigma). Pools of zebrafish embryos $(n=30)$ were collected stored dry $\left(-80^{\circ} \mathrm{C}\right)$ at gastrula $(6 \mathrm{hpf})$ and segmentation stage (15 hpf). Lysates from non-injected control embryos and those injected with myc-tagged $A R F 3^{W T}, A R F 3^{K 127 E}$ and $A R F 3^{L 12 V ; D 67 V}$ were obtained by syringe homogenization in lysis buffer (Tris $\mathrm{HCl} 10$ mM pH 7.4; EDTA 2 mM; NaCl 150 $\mathrm{mM}$; Triton X-100 1\% with 1X protein inhibitors cocktail (Roche) and equal amounts of protein extracts $(40 \mu \mathrm{g})$ were separated on a $12 \%$ Sodium dodecyl sulfate (SDS)polyacrylamide gel. The total protein concentration was determined by the Bradford assay (Bio-Rad) using Cary 100 UV-Vis (Agilent Technologies). After electrophoresis, the proteins were transferred to PVDF membrane (Bio-Rad) using a wet transfer system (Biorad). Blots were blocked with 5\% non-fat milk powder (Biorad) or bovine serum albumin (Sigma-Aldrich) in PBS containing $0.1 \%$ Tween-20 overnight at $4^{\circ} \mathrm{C}$ constantly shaking and incubated with primary antibody in blocking solution. The following primary antibodies were used: mouse monoclonal anti-myc (Cell Signaling, 
Dominantly acting variants in ARF3 have disruptive consequences on Golgi integrity and cause microcephaly recapitulated in zebrafish

Fasano, Muto, Radio, et al.

652 dilution 1:1000), rabbit polyclonal anti-GAPDH (Genetex, dilution 1:1000). Following 653 washes in PBST $0.1 \%$, membranes were incubated with anti-mouse and anti-rabbit654 HRP-conjugated secondary antibodies (1:3000) (Sigma). Immunoreactive proteins 655 were detected by an enhanced chemiluminescence (ECL) detection kit (Thermo Fisher) according to the manufacturer's instructions, and an Alliance Mini HD9 was used for chemiluminescence detection (Uvitec).

\section{$658 \quad$ ARF3 protein stability assays}

659 COS-1 cells were seeded at $3 \times 10^{5}$ in 6 -well plates and the following day were 660 transfected with WT or mutant myc-tagged ARF3 expression constructs for 24 hours. 661 A subset of transfected cells was then treated with proteasome inhibitor MG132 (100 $\mu \mathrm{M})$ or with the autophagy inhibitor bafilomycin A1 (200 nM) (Sigma-Aldrich) for 6 663 hours.

\section{ARF3 activation assay}

665 COS-1 cells $\left(1 \times 10^{6}\right)$ were seeded in $100 \mathrm{~mm}$ petri dishes and transfected with myc666 tagged ARF3 expression constructs. Twenty-four hours after transfection, cells were

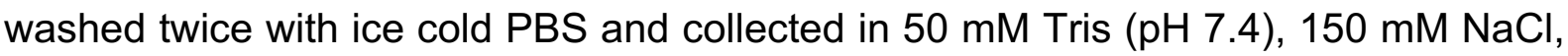
$10 \mathrm{mM} \mathrm{MgCl}_{2}, 10 \%$ Glycerol, $1 \% \mathrm{NP}-40$ with proteases and phosphatase inhibitors (Sigma-Aldrich). Cell lysates were further subjected to pull-down using GGA3-

670 conjugated agarose beads (Cell Biolabs) and incubated at $4{ }^{\circ} \mathrm{C}$ for $60 \mathrm{~min}$. For

671 immunoblotting analyses, pulled down samples and whole cell lysates were combined 672 with a $2 x$ sample buffer and denatured at $95{ }^{\circ} \mathrm{C}$ for $5 \mathrm{~min}$. Samples were then separated by SDS-PAGE and incubated with anti-myc and anti- $\beta$-tubulin antibodies.

674 GTP-bound protein level was detected by an ECL detection kit (Thermo Fisher). 
Dominantly acting variants in ARF3 have disruptive consequences on Golgi integrity and cause microcephaly recapitulated in zebrafish

Fasano, Muto, Radio, et al.

675

676

677

678

679

680

681

682

683

684

685

686

687

688

689

690

691

692

693

694

695

696

697

698

ARF3 protein localization and Golgi morphology assessment in fixed COS-1 cells

COS-1 cells $\left(30 \times 10^{3}\right)$ were seeded in 24-well cluster plates onto 12-mm cover glasses and transfected with WT or mutant mCherry-tagged ARF3 expression constructs for 24 hours. Cells were then fixed with $3 \%$ paraformaldehyde for 30 minutes at $4{ }^{\circ} \mathrm{C}$, followed by permeabilization with $0.5 \%$ Triton $\mathrm{X}-100$ for 5 minutes at room temperature. Cells were stained with mouse monoclonal anti-Golgin 97 antibody (1:50, Abcam) for 1 hour at room temperature, rinsed twice with PBS and incubated with Alexa Fluor 488 goat anti-mouse secondary antibody (1:200, Molecular Probes) for 1 hour at room temperature. After staining, coverslips were mounted on slides by using Vectashield Antifade mounting medium (Vector Laboratories) containing $1.5 \mu \mathrm{g} / \mathrm{ml}$ DAPI (Sigma). Images were acquired using Olympus Fluoview FV1000 confocal microscope using $60 x / 1.42$ oil objective and signals from different fluorescent probes were taken in sequential scanning mode. Cells were screened for incidence of Golgi phenotype.

\section{Time-lapse imaging of Golgi dynamics in COS-1 cells expressing ARF3}

For live imaging, COS-1 cells $\left(10 \times 10^{4}\right)$ were seeded into $\mu$-dishes $35 \mathrm{~mm}$ (Ibidi) 24 hours before transfection. The day after, cells were co-transfected with WT or mutant mCherry-tagged $A R F 3$ and EGFP-GalT constructs. Four hours posttransfection, time-lapse acquisitions were performed with a Leica TCS-SP8X confocal microscope (Leica Microsystems) with a PIApo CS2 20x/0.75 objective. Zreconstructions of serial single optical sections were obtained every 15 min and carried out with a $1024 \times 1024$ format, scan speed of $600 \mathrm{~Hz}$, a zoom magnification up to 1.5 and z-step size of $0.7 \mu \mathrm{m}$. Live imaging of samples was performed simultaneously using the Mark \& Find mode of the LAS X software. Time-lapse microscopy was 
Dominantly acting variants in ARF3 have disruptive consequences on Golgi integrity and cause microcephaly recapitulated in zebrafish

Fasano, Muto, Radio, et al.

699

700

701

702

703

704

705

706

707

708

709

710

711

712

713

714

715

716

717

718

719

720

721

performed with a stage incubator (OkoLab) allowing to maintain stable conditions of temperature, $\mathrm{CO}_{2}$ and humidity during live cell imaging.

\section{Zebrafish husbandry}

Zebrafish NHGRI-193 and Tg(NBT: dsRed) ${ }^{94}$ were cultured following standard protocols ${ }^{95}$. Fish were housed in a water circulating system (Tecniplast) under controlled conditions (light/dark 14:10, $28{ }^{\circ} \mathrm{C}, 350-400$ uS, pH 6.8-7.2) and fed daily with dry and live food. All experiments were approved by the Italian Ministry of Health (23/2019-PR).

\section{Zebrafish mRNA microinjection}

Injection of in vitro synthesized capped mRNAs encoding myc-tagged ARF3 (7.5 and 15 pg), mCherry-tagged ARF3 (15 pg), mKOFP-CAAX (15 pg), H2A-mCherry (15 pg), EGFP-GalT (15 pg), EGFP-CAAX (15 pg) and EGFP-GalT (50pg) was performed in one-cell stage zebrafish embryos using FemtoJet $4 x$ microinjection system (Eppendorf). Injected embryos were cultured under standard conditions at $28{ }^{\circ} \mathrm{C}$ in fresh $\mathrm{E}_{3}$ medium and for each batch, non-injected fish were used as controls together with fish injected with the WT form of ARF3 mRNA. Embryos were monitored every day and survival rate was analyzed at 24 and 48 hours post-fertilization (hpf).

\section{Zebrafish body axis, notochord and head phenotyping}

Embryos were screened for gross phenotype penetrance classified as normal, mild and severe at $24 \mathrm{hpf}$. For detailed analysis, not-injected controls and injected fish at 12 and $15 \mathrm{hpf}$ (for body axis), $24 \mathrm{hpf}$ (for notochord) and $48 \mathrm{hpf}$ (for head size) were embedded in $2 \%$ low melting agarose dissolved in $\mathrm{E}_{3}$ medium. Bright-field images were acquired at Leica M205FA microscope with 0.63x magnification (Leica Microsystems). 
Dominantly acting variants in ARF3 have disruptive consequences on Golgi integrity and cause microcephaly recapitulated in zebrafish

Fasano, Muto, Radio, et al.

722 These parameters were assessed: i) angle between the antero-posterior ends (body

723 axis); ii) number of notochord curvatures and degree of the notochord angles (plotted

724 in a Rose diagram using Oriana)96; iii) head size measured by the area surface between the rostral most part of the head and the optic vesicle.

Whole-mount immunofluorescence for acetylated a-tubulin and HuC/Elav in zebrafish embryos

Whole-mount samples were fixed in $4 \%$ paraformaldehyde (Thermo Fisher), washed in PBS-Triton 0.8\% (PBSTr 0.8\%), permeabilized with proteinase $\mathrm{K}$ treatment $(1 \mu \mathrm{g} / \mathrm{ml})$ and incubated in $2 \%$ blocking reagent (Roche) for 2 hours. Samples were then incubated with the primary antibody in $2 \%$ blocking reagent overnight at $4{ }^{\circ} \mathrm{C}$ (anti acetylated a-tubulin, 1:500, Sigma-Aldrich). After several washes in PBSTr 0.8\%, samples were incubated with the Alexa Fluor 488 goat anti-mouse secondary antibody (1:1000, Thermo Fisher) overnight at $4{ }^{\circ} \mathrm{C}$ with gentle shaking. Whole-mount larval samples were mounted in $90 \%$ glycerol. Dorsal z-stacks (volumes) of embryos stained for acetylated a-tubulin were acquired with a Leica TCS-SP8X confocal microscope with PIApo CS2 20x/0.75 objective scanning with $1024 \times 1024$ format, speed of 400 $\mathrm{Hz}$ and z-step size of $2 \mu \mathrm{m}$. Embryos stained for HuC/Elav were imaged as above or using Olympus FV1000, objective 20x/0.75 dry, with same parameters.

Zebrafish live brain volume imaging and 3D rendering from $T g(N B T: d s R e d)$ fish

741 Live confocal acquisitions from $48 \mathrm{hpf}$ injected $\mathrm{Tg}(\mathrm{NBT}$ :dsRed) fish embedded in $2 \%$ 742 low melting agarose in $\mathrm{E}_{3}$ medium were obtained with Leica Stellaris 5 confocal

743 microscope using hybrid detectors and keeping minimal laser power. Scans were 744 obtained with $512 \times 512$ resolution, $400 \mathrm{~Hz}$. Live z-stacks were acquired with Fluotar $74525 x / 0.95$ water-immersion objective and with a z-step size of $2.5 \mu \mathrm{m}$. Volumetric brain 
Dominantly acting variants in ARF3 have disruptive consequences on Golgi integrity and cause microcephaly recapitulated in zebrafish

Fasano, Muto, Radio, et al.

746

747

reconstructions from representative $48 \mathrm{hpf}$ injected $\mathrm{Tg}(\mathrm{NBT}: \mathrm{dsRed}$ ) fish were obtained using 3D Volume (Blend model) and Surfaces rendering functions of Imaris (Bitplane), employing the same parameters for the different individuals.

\section{Zebrafish brain volume analysis}

Anterior brain volume was measured on both live $\operatorname{Tg}(N B T: d s R e d)$ embryos and fixed specimens stained with antibodies against HuC/Elav and acetylated a-tubulin. A region of interest (ROI) comprising the most anterior part of the $N B T: d s R e d+$ neurons and the cerebellum was selected along the whole z-stacks of the confocal 3D data using Fiji analysis tools. Image data were binarized employing Threshold/Otsu and B\&W/Dark background algorithms. For every z-stack layer the surface area measurement was extrapolated using a custom-made macro. Volumetric measurements were obtained taking into account the $x$ Z-step value.

\section{Whole-mount in situ hybridization of Krox20 and MyoD mRNA}

The fragments of Krox-20 and MyoD cDNA used for riboprobe synthesis were amplified from a zebrafish cDNA preparation by PCR using One Taq DNA polymerase (NEB New England Biolabs) and the primers listed in Supplementary Table 10. The PCR fragments were cloned into pGEM-T Easy vector (Promega) and sequences were confirmed by DNA sequencing. The digoxigenin-labeled antisense riboprobes were synthesized by in vitro transcription with DIG RNA labeling kit SP6/T7 (Roche). In situ hybridization analysis in whole-mount zebrafish embryos at $15 \mathrm{hpf}$ was performed as previously described ${ }^{97}$. Briefly, samples were permeabilized with proteinase $\mathrm{K}$ treatment $(1 \mu \mathrm{g} / \mathrm{ml})$ for 2 minutes, pre-incubated in $2 \%$ blocking reagent (Roche) and incubated with riboprobes $(2 \mathrm{ng} / \mu \mathrm{l})$ in hybridization mix ( $50 \%$ formamide, $1.3 x \mathrm{SSC}$, $100 \mathrm{~g} / \mathrm{ml}$ heparin, $50 \mu \mathrm{g} / \mathrm{ml}$ yeast RNA, 0.2\% Tween-20, 0.5\% CHAPS, $5 \mathrm{mM}$ EDTA 
Dominantly acting variants in ARF3 have disruptive consequences on Golgi integrity and cause microcephaly recapitulated in zebrafish

Fasano, Muto, Radio, et al.

770

771

772

773

774

775

776

777

778

779

780

781

782

783

784

785

786

787

788

789

790

791

792

793

$\mathrm{pH} 8)$ at $65^{\circ} \mathrm{C}$ for at least $15 \mathrm{hrs}$. Afterwards, samples were rinsed with scalar dilutions of SSC solutions and incubated with anti-alkaline phosphatase (AP)-conjugated antibody (1:5000, Roche) for 2 hours at room temperature. Chromogenic staining was developed via BM Purple substrate (Roche) according to manufacturer's instructions. Specimens were mounted in $90 \%$ glycerol and dorsal images were acquired from Olympus TH4-200 microscope (Olympus Life Science) with 10x objective.

\section{Confocal live imaging of zebrafish embryos during gastrulation}

For in vivo imaging, embryos at mid-gastrula stage were embedded in $2 \%$ low melting agarose dissolved in $\mathrm{E}_{3}$ medium. 4D fluorescent data were acquired using Leica TCSSP8X confocal microscope using hybrid detectors, keeping minimal laser power. Scans were obtained in a 1024 x 1024 format, with a speed of $400 \mathrm{~Hz}$. Fluorochromes unmixing was performed by acquisition of automated-sequential collection of multichannel images, in order to reduce spectral crosstalk between channels, and the same setting parameters were used for all examined samples. For ARF3 localization and Golgi detection sequential confocal images were acquired with Fluotar 25x/0.95 waterimmersion objective with a z-step size of $2.5 \mu \mathrm{m}$. Gastrulation time-lapses were acquired with a PIApo CS2 20x/0.75 objective. Z-reconstructions of serial single optical sections were obtained every 30 min with a z-step size of $3 \mu \mathrm{m}$. Embryo live imaging was performed simultaneously using the Mark \& Find mode of the LAS X software.

\section{Trans-Golgi fragmentation analysis in live zebrafish embryos}

Fragmented trans-Golgi morphology was assessed from single confocal images of the animal pole of at $12 \mathrm{hpf}$ embryos injected with $A R F 3^{W T}$ and $A R F 3^{K 127 E}$ GalT-GFP and mKOFP-CAAX mRNA. Trans-Golgi morphology (GalT-GFP+) in each cell was scored as "ribbon" if it displayed a recognizable circular and compact structure or "puncta" if it 
Dominantly acting variants in ARF3 have disruptive consequences on Golgi integrity and cause microcephaly recapitulated in zebrafish

Fasano, Muto, Radio, et al.

794 displayed a clear fragmented pattern. Circa 30-40 cells per embryo were counted, $n=$ $7954\left(\mathrm{ARF}^{\mathrm{WT}}\right)$ and $\mathrm{n}=5\left(\mathrm{ARF} 3^{\mathrm{K} 127 \mathrm{E}}\right)$ embryos. Fisher's exact test in a $2 \times 2$ contingency 796 table (puncta vs. ribbon) $\left({ }^{* * * *} p<0.0001\right)$ is used to assess statistical significance.

\section{Statistical analysis and Image processing}

798

Data were analyzed independently by at least two researchers and statistical assessments were performed using GraphPad Prism. Log-rank (Mantel-Cox) test was used to assess survival in zebrafish mutants. For phenotype penetrance assessment

801 Fisher's exact test in a 2x2 contingency table was used, performed as pairwise 802 statistical comparisons across experimental conditions (normal versus mild or severe 803 in vivo and compact versus fragmented Golgi morphology phenotype for in vitro 804 analyses). Normality tests (Anderson-Darling, D’Agostino \& Pearson, Shapiro-Wilk and Kolmogorov-Smirnov tests) were run to assess normal distribution of the data. 806 Parametric data with more experimental groups were analyzed with Anova test, non807 parametric data with Kruskal-Wallis test and specific post hoc tests were always used 808 as indicated in the figure legends. Whenever multiple measurements in zebrafish were performed on the same individual (e.g., different cells from the same embryo), analysis with a mixed-effects model were used to take account of resampling from the same

811 cluster (embryo). All the analyses were two-tailed. Outliers identification were 812 assessed by ROUT method $(\mathrm{Q}=1 \%)$. An overview of the statistical analysis for each 813 dataset is shown in Supplementary Table 11. Raw images were analyzed with Fiji ${ }^{98}$, 814 LAS X Life Science imaging software (Leica Microsystem), FV10-ASW software, 815 Olympus CellSens Standard imaging software (Olympus Life Science) and Imaris 816 (Bitplane) and processed using Photoshop or Illustrator (Adobe Systems Incorporated) 
Dominantly acting variants in ARF3 have disruptive consequences on Golgi integrity and cause microcephaly recapitulated in zebrafish

Fasano, Muto, Radio, et al.

817 for figure assembly. Brightness and contrast were adjusted equally across the whole 818 image.

819 Supplemental data description

820 The article includes the following supplemental data:

821 Supplementary Methods and clinical reports, 11 figures, 11 tables and 1 video (.mp4).

822 Acknowledgments

823 H2A-mCherry:pDest and GFP-CAAX:pDest plasmids were kindly provided by Dr. 824 Mette Handberg-Thorsager (Max Planck Institute of Molecular Cell Biology and 825 Genetics, Dresden, MPI-CBG, Germany). We acknowledge Cineca ELIXIR-IIB for 826 computing resources. This work was supported, in part, by Fondazione Bambino Gesù 827 (Vite Coraggiose to M.T.), Italian Ministry of Health (CCR-2017-23669081 and RCR828 2020-23670068_001 to M.T; RF-2018-12366931 to F.C.R., G.C. and B.D.; Ricerca 829 Corrente 2021 to A.L., S.C. and M.T., and RF-2013-02355240 to R.G.), Italian Ministry 830 of Research (FOE 2019 to M.T.), the Tuscany Region Call for Health 2018 (DECODE 831 EE, to R.G.), and the European Union Seventh Framework Programme (DESIRE 832 [602531] to R.G.).

\section{Authors'contribution}

834 G.F. designed and performed the in vivo experiments and contributed to write the manuscript. V.M. designed and performed the in vitro experiments and contributed to write the manuscript. F.C.R. coordinated the clinical data collection and phenotyping, analyzed the clinical data and contributed to write the manuscript. L.A.C., S.P. and

838 S.C. performed the confocal scanning experiments. M.V. performed the in situ 839 hybridization assays. A.Z., A.V., F.P., S.P., A.B., and M.I. generated and analyzed the 
Dominantly acting variants in ARF3 have disruptive consequences on Golgi integrity and cause microcephaly recapitulated in zebrafish

Fasano, Muto, Radio, et al.

840 genomic data. G.C., I.G.P. and B.C. performed the structural analyses and molecular

841 dynamics simulations. M.B., C.B., D.M., A.S., M.M., M.V.G., A.B., R.G., A.S. and B.D.

842 identified the patients, and collected and analyzed the clinical data. A.L. and M.T.

843 conceived, designed, supervised the project, analyzed the data, and wrote the

844 manuscript.

845

846 Declaration of Interests

847 The authors declare no competing interests.

848 Data availability

849 The data generated in this work are available upon request from the corresponding 850 authors. 
a

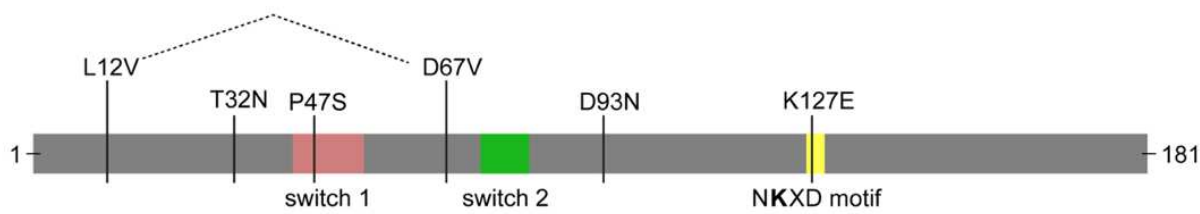

b

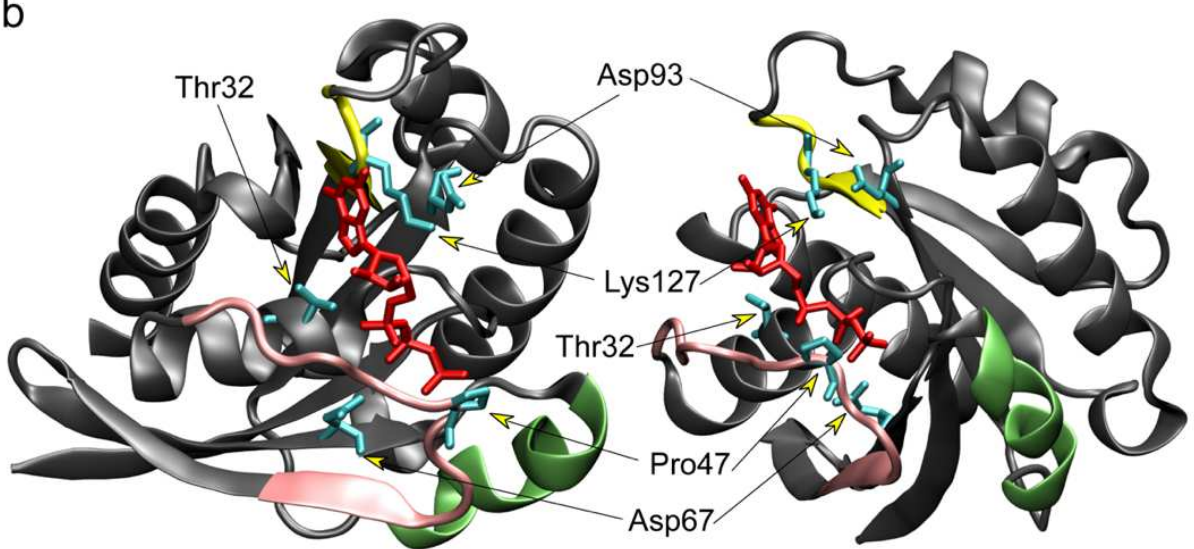

C

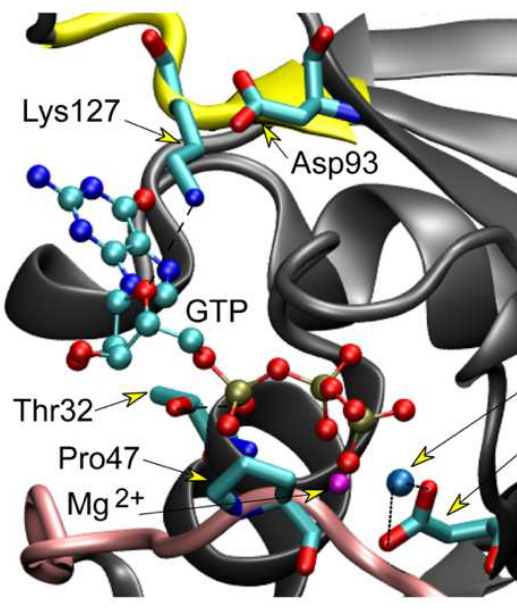

d
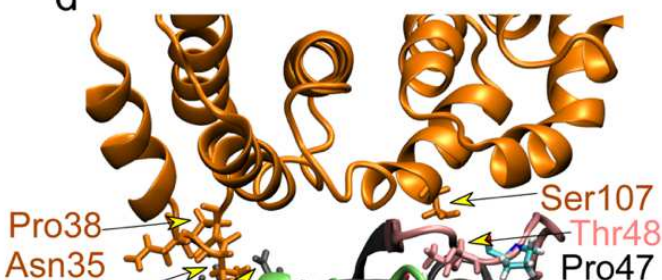

inter or

Thr37 , 70 , WAT ${ }_{\text {Asp67 }}^{\text {Arg19 }}{ }^{2}$

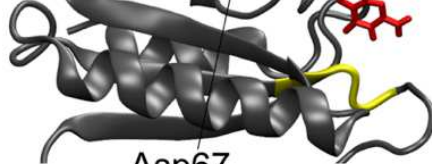

Asp67

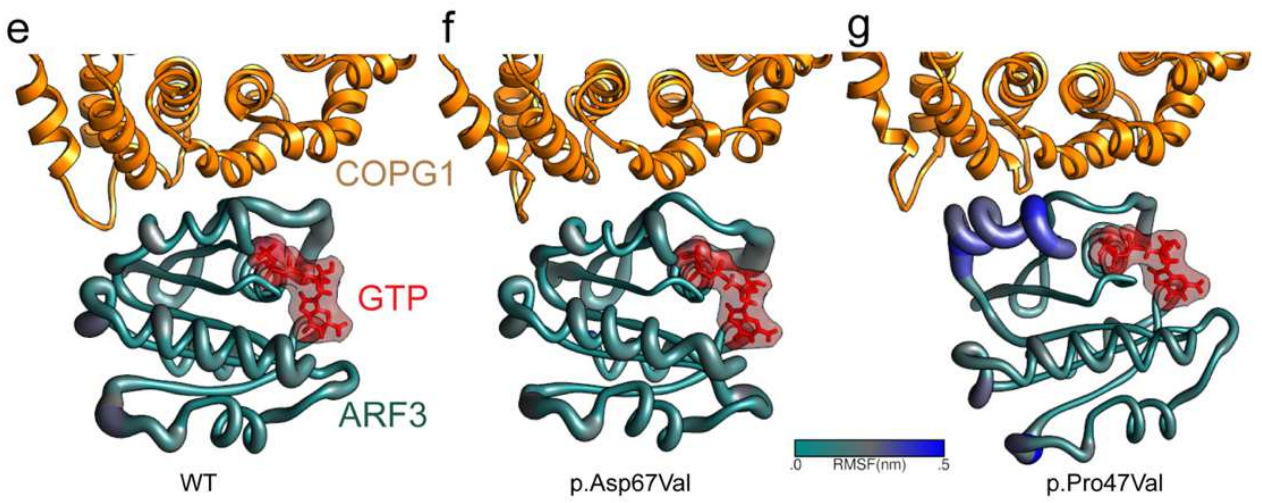

852

p.Asp67Val

p.Pro47Val

853 Figure 1. Structural organization of ARF3, location of mutated residues and

854 molecular dynamics analyses. (a) Domain organization of ARF3 excluding the 
Dominantly acting variants in ARF3 have disruptive consequences on Golgi integrity and cause microcephaly recapitulated in zebrafish

Fasano, Muto, Radio, et al.

855

unstructured $C$-terminal tail. Switch 1 , switch 2 and the NKXD fingerpoint motif are highlighted in pink, green and yellow, respectively. The variants identified in affected subjects are also reported; (b-c) 3D structure in two different orientations of GTPbound ARF3 interacting with the MARTX toxin (PDB 6ii6). Side chains of the ARF3 residues mutated in the affected subjects and GTP are in cyan and red, respectively. Main chain of residues belonging to switch 1 , switch 2 and NKXD fingerpoint motif are colored as above; (d) Enlargement of the ARF3 GTP binding pocket with the five mutated residues. The direct hydrogen bond between the $\mathrm{N}$ atom in the Lys127 lateral chain and the oxygen atom of the GTP ribose ring is highlighted in dashed line. The $\mathrm{Mg}^{2+}$ ion is colored in magenta, while the oxygen atom of the water molecule, mediating the interaction between Asp67 and the manganese ion, is shown in light blue color. The two hydrogen bonds between Asp67 and the water molecule are highlighted with dotted lines. (e) Homology model of GTP-bound ARF3 interacting with the cytosolic coat protein complex COPG1-COPZ1 (PDB: 3TJZ) validated by a 500-ns molecular dynamics (MD) simulation. The region of contact between ARF3 and COPG1 (orange color) is shown in e. (f-h) MD simulations of wild-type (f), p.Asp67Val (g) and p.Pro47Ser (h) ARF3 complexed with COPG1-COPZ1. Residues involved in the contact are shown with their side chain and colored as the respective protein/region. ARF3 backbone is represented with a diameter proportional to its per-residue fluctuations (RMSF). 
Dominantly acting variants in ARF3 have disruptive consequences on Golgi integrity and cause microcephaly recapitulated in zebrafish

Fasano, Muto, Radio, et al.

a

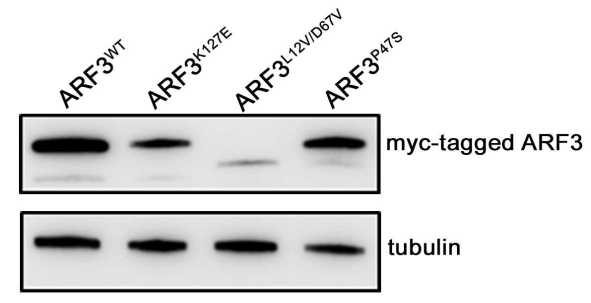

b

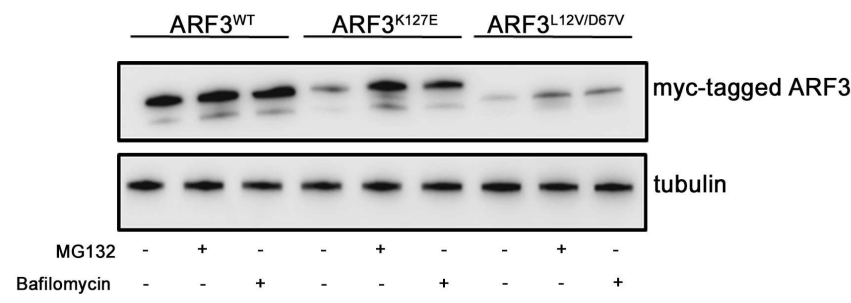

C

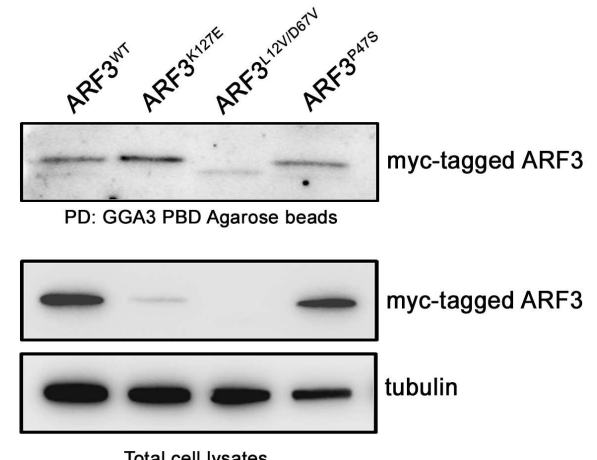

Total cell lysates
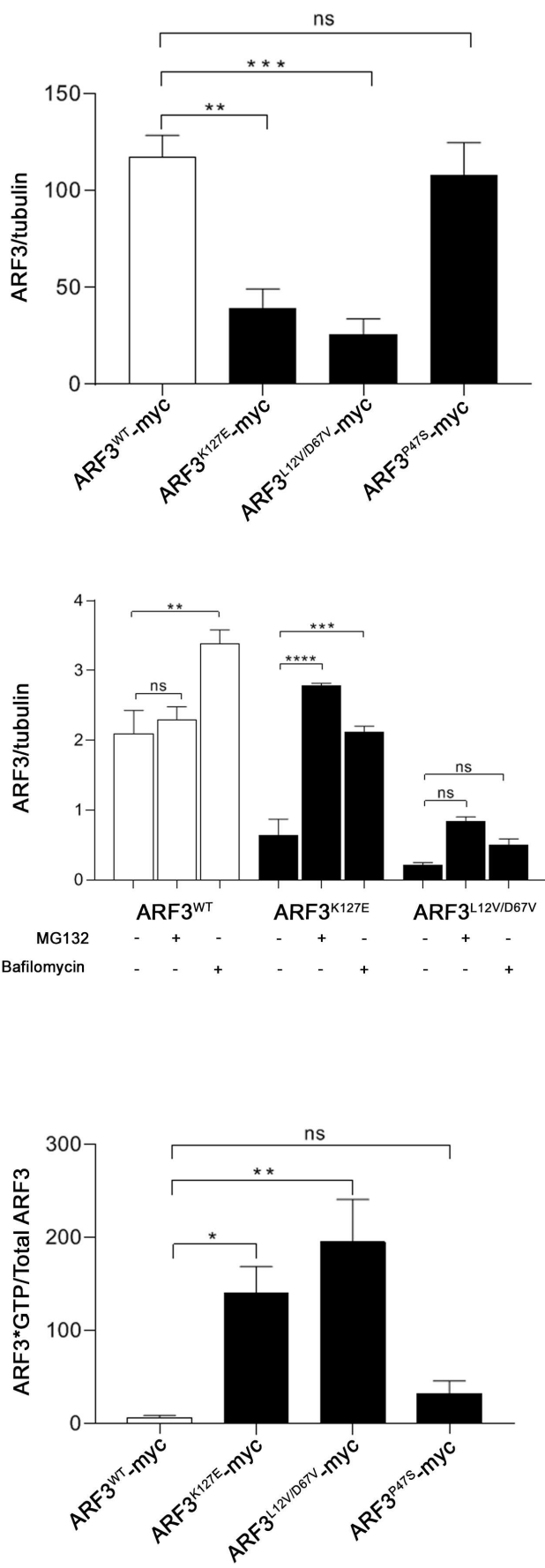
protein in COS-1 cells. (a) Expression levels of each mutant myc-tagged ARF3

878 protein assessed in transiently transfected COS-1 cells via immunoblot. (b) Western

879 blot analysis shows the protein levels of WT ARF3 and ARF3 ${ }^{\mathrm{K} 127 \mathrm{E}}$ and ARF3 ${ }^{\mathrm{L} 12 \mathrm{~V} ; \mathrm{D} 67 \mathrm{~V}}$

880 mutants in transfected COS-1 cells, basally and after treatment with MG132 (100 $\mu \mathrm{M})$ 
Dominantly acting variants in ARF3 have disruptive consequences on Golgi integrity and cause microcephaly recapitulated in zebrafish

Fasano, Muto, Radio, et al.

881 and bafilomycin A1 (200 nM) for six hours. (c) Pull-down assay using GGA3882 conjugated beads shows ARF3 activation in COS-1 cells transiently expressing WT or 883 mutant myc-tagged ARF3 proteins. Active and total ARF3 levels are monitored using 884 anti-myc antibody. Representative blots and mean \pm standard error (SEM) of three 885 independent experiments are shown. Statistical differences are obtained by one-way 886 Anova (a,c) followed by Dunnet post hoc test and two-way Anova (b) (ns = not 887 significant, $\left.{ }^{*} p<0.05,{ }^{* *} p<0.01,{ }^{* * *} p<0.001,{ }^{* * * *} p<0.0001\right)$. For the ARF3 ${ }^{L 12 V ; D 67 V}$ 888 mutant the statistical comparison between no treatment (-) and treatment with MG132 889 results in $p=0.07$ indicating an overall trend towards increased expression levels of the mutant ARF3 following the treatment. 
Dominantly acting variants in ARF3 have disruptive consequences on Golgi integrity and cause microcephaly recapitulated in zebrafish

Fasano, Muto, Radio, et al.
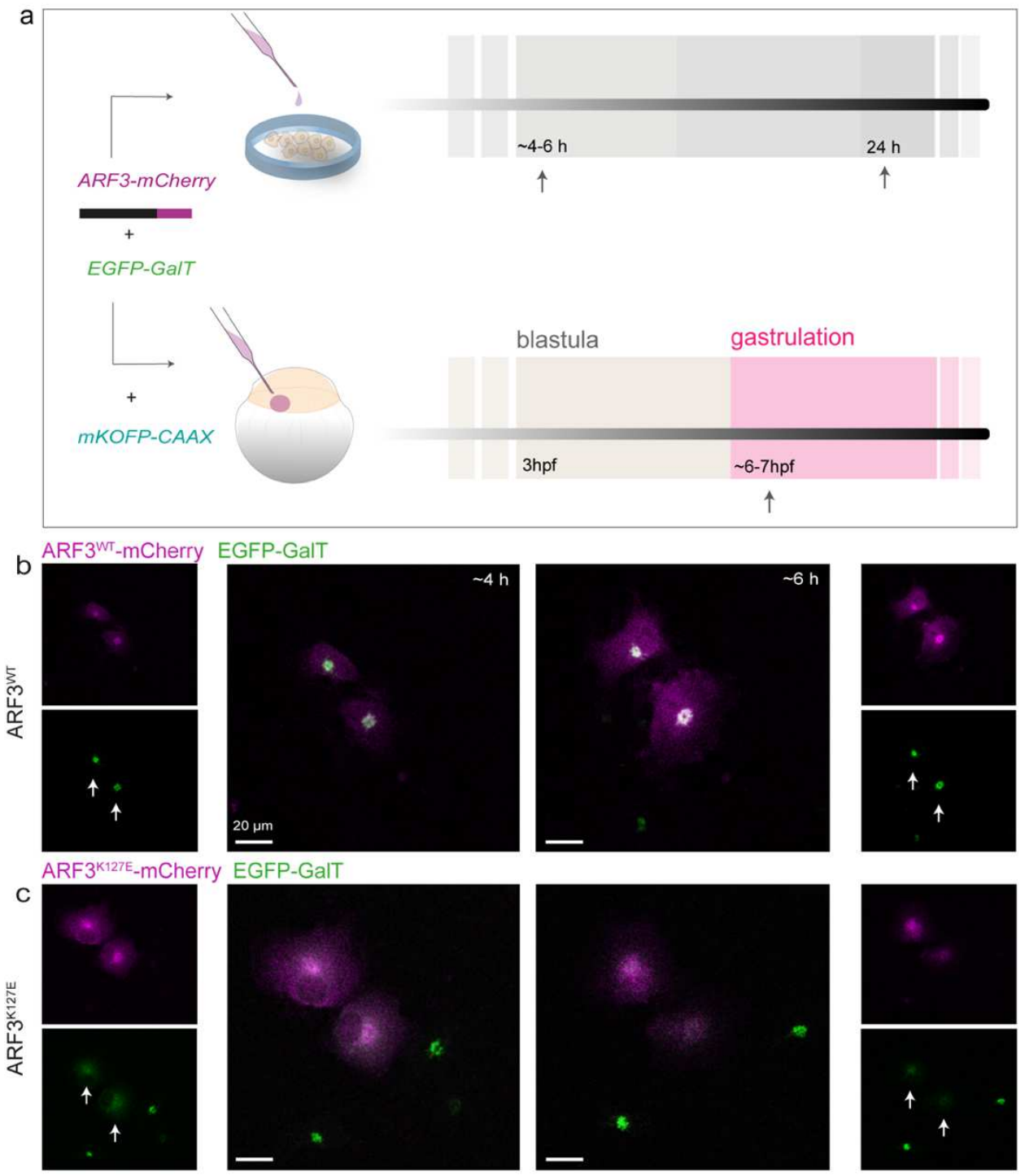

ARF3 ${ }^{W T}$-mCherry EGFP-GalT
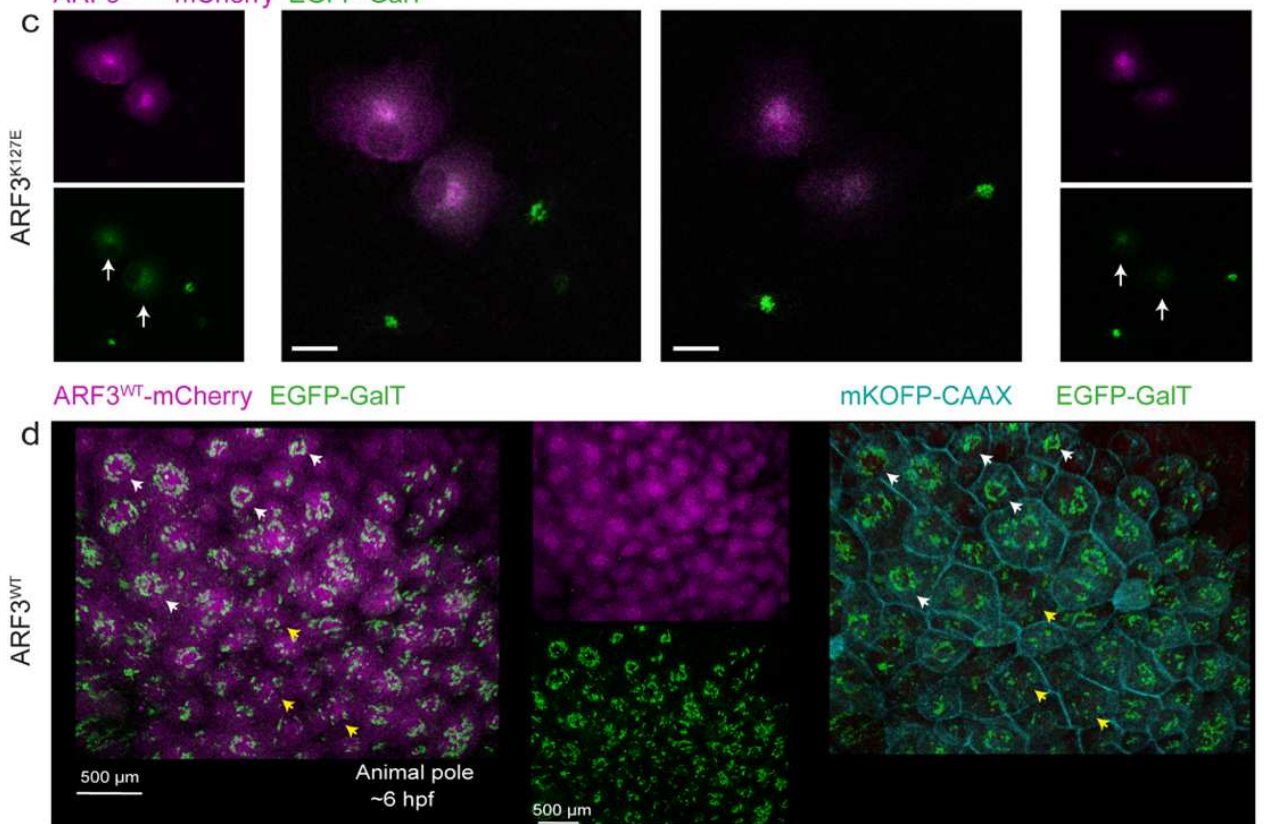

MKOFP-CAAX EGFP-GalT

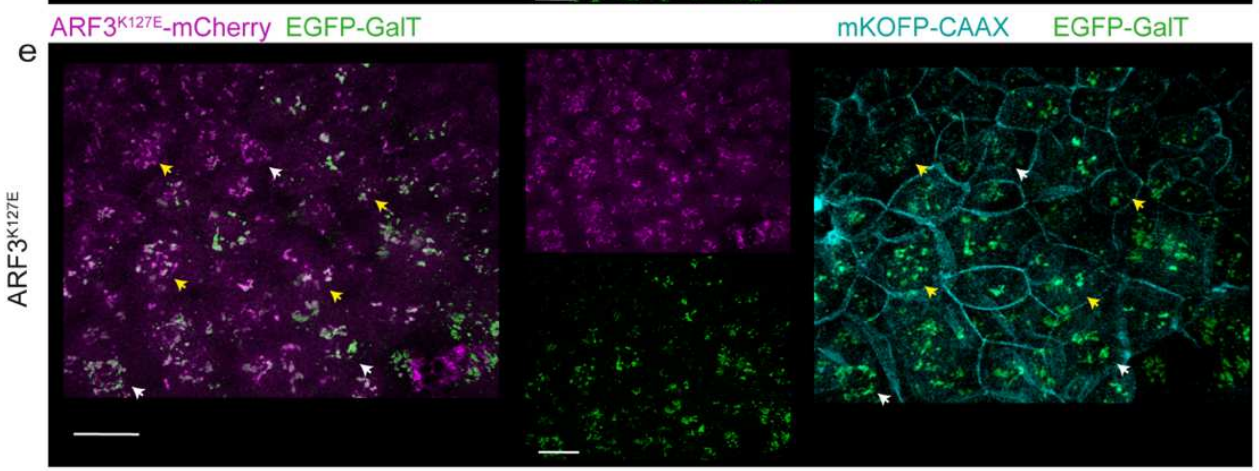


Dominantly acting variants in ARF3 have disruptive consequences on Golgi integrity and cause microcephaly recapitulated in zebrafish

Fasano, Muto, Radio, et al.

892

893

894

895

896

897

898

899

900

901

902

903

904

905

906

907

908

909

910

Figure 3. Trans-Golgi fragmentation in cells and zebrafish embryos expressing the mutant mCherry-tagged ARF3 ${ }^{\mathrm{K} 127 \mathrm{E}}$. (a) Schematic representation of the experimental set up in both in vitro and in vivo systems. COS-1 cells are transfected with DNA constructs expressing WT and mutant ARF3-mCherry (magenta) and EGFPGalT (trans-Golgi maker, green) and analyzed by live confocal microscopy between 46 hours post transfection. Zebrafish embryos are injected at 1 cell stage with WT and mutant ARF3-mCherry and EGFP-GalT mRN. mKOFP CAAX mRNA is used as membrane marker (cyan). Animals are analyzed by live confocal microscopy during gastrulation ( 6-7 hpf). (b, c) Maximum intensity projections of representative confocal images from a time-lapse experiment (Video S1) performed in transfected COS-1 cells at $15 \min (\sim 4$ hours post transfection) and 120 min later ( 6 hours post transfection) from the start of the time-lapse experiment. The images show diffused EGFP-GalT signal (trans-Golgi fragmentation) in $\mathrm{ARF} 3^{\mathrm{K} 127 \mathrm{E}}$ over time (white arrows). Scale bar $=$

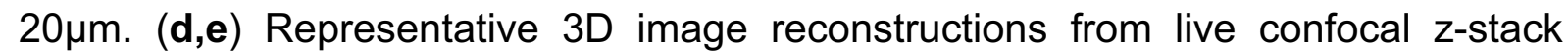
acquisitions of the animal pole in developing zebrafish embryos expressing ARF $3^{W T}$ and $\mathrm{ARF}^{\mathrm{K} 127 \mathrm{E}}$ at the mid-gastrulation stage (between 6-7 hpf). White arrowheads indicate a compact trans-Golgi morphology surrounding the nucleus ("ribbon") in the envelope layer cells. Yellow arrowheads indicate cells showing "punta" morphology of the TG dispersed throughout the cytosol. Scale bar $=500 \mu \mathrm{m}$. 

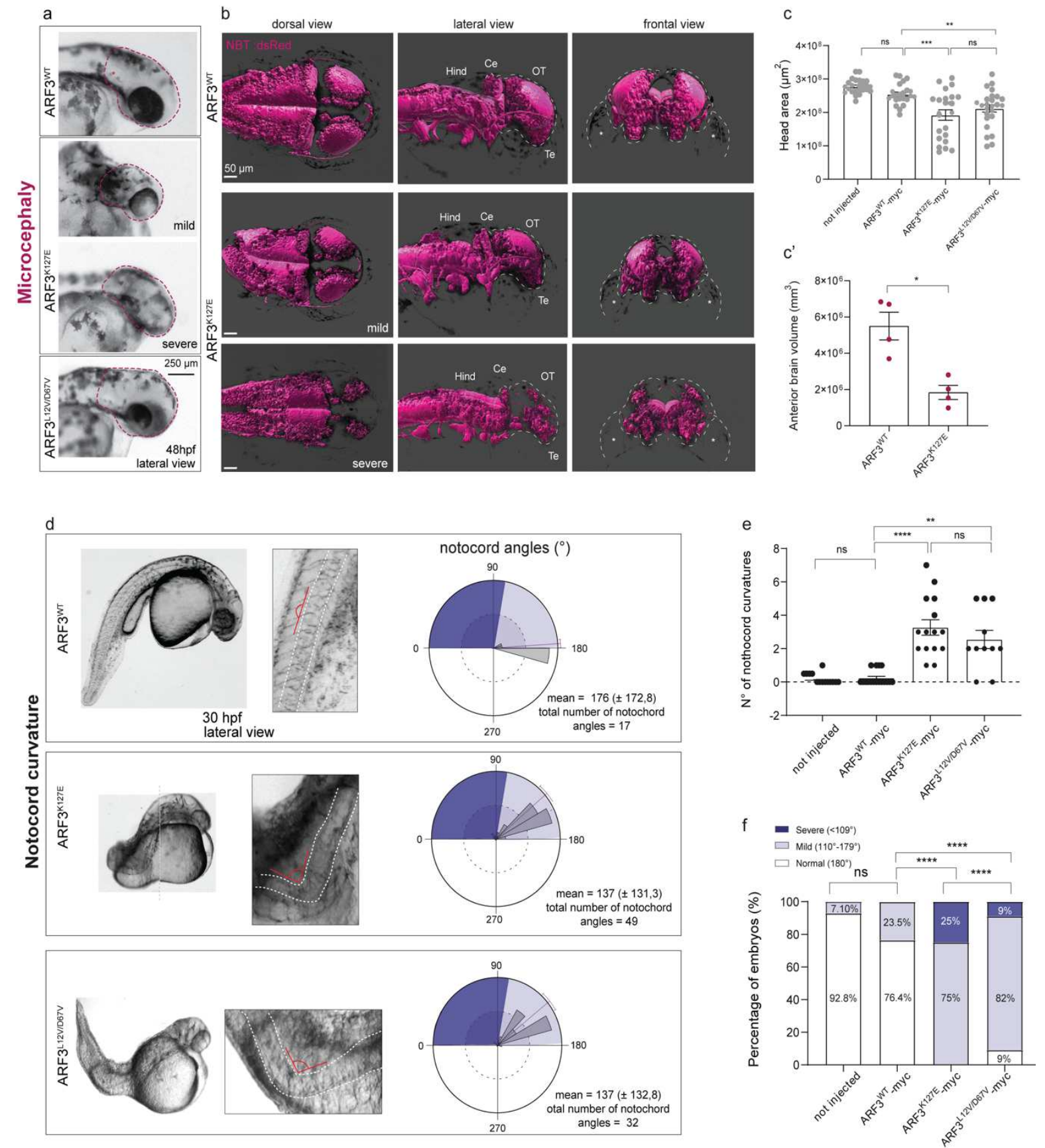

912 Figure 4. Zebrafish embryos expressing ARF3 mutant proteins show

913 microcephaly and axial defects with notochord curvatures of variable severities.

914 (a) Representative bright field images of the head (purple dashed line) in fish injected

915 with ARF3 WT or ARF3 ${ }^{\mathrm{K} 127 \mathrm{E}}$ and $\mathrm{ARF} 3^{\mathrm{L} 12 \mathrm{VD} 67 \mathrm{~V}}$ mutants at $48 \mathrm{hpf}$, scale bar $=250 \mu \mathrm{m}$.

916 (b) Volumetric reconstructions from live confocal acquisitions of whole brains in $48 \mathrm{hpf}$

$917 \operatorname{Tg}(\mathrm{NBT}: d s R e d)$ fish injected with $\mathrm{ARF} 3^{\mathrm{WT}}$ and $\mathrm{ARF}{ }^{\mathrm{K} 127 \mathrm{E}}$ show examples of mild and 
Dominantly acting variants in ARF3 have disruptive consequences on Golgi integrity and cause microcephaly recapitulated in zebrafish

Fasano, Muto, Radio, et al.

918 severe microcephaly, scale bar $=50 \mu \mathrm{m}$. Te: telencephalon, OT: optic tectum, ce:

919 cerebellum, hind: hindbrain. (c) Head area (indicated by the purple dashed contour in

a) in $\operatorname{ARF}^{\mathrm{K} 127 E}(n=22)$ and $\operatorname{ARF}^{\operatorname{L12V;D67V}}(n=25)$ not injected controls $(n=25)$ and

$921 \operatorname{ARF}^{\mathrm{WT}}(\mathrm{n}=26)$ expressing fish. (c') Brain volume in $\operatorname{Tg}(\mathrm{NBT}$ :dsRed) fish at 48hpf, $\mathrm{n}$

$922=4$. (d) Representative bright filed images of notochord curvatures (red angle

923 calculated on single notochords), classified as normal (angle $\left.=180^{\circ}\right)$, mild $\left(179^{\circ}\right.$

$924>$ angle $\left.>110^{\circ}\right)$ and severe $\left(109^{\circ}>\right.$ angle $\left.>0^{\circ}\right)$, in fish expressing ARF $3^{\mathrm{WT}}, \mathrm{ARF} 3^{\mathrm{K} 127 \mathrm{E}}$ and

$925 \mathrm{ARF}^{\mathrm{L} 12 \mathrm{~V} ; \mathrm{D} 67 \mathrm{~V}}$ at $30 \mathrm{hpf}$. The rose diagrams show the distributions of angles for each

set of notochordal observations. (e) Number of notochord curvatures in embryos

expressing mutant or WT ARF3. (f) Incidence of embryos showing notochord

curvatures. In e and $f \mathrm{n}=14,17,15,11$ for not injected, ARF3 ${ }^{\mathrm{WT}}, \mathrm{ARF} 3^{\mathrm{K} 127 \mathrm{E}}$ and

$929 \mathrm{ARF}^{\mathrm{L} 12 \mathrm{VD} 67 \mathrm{~V}}$, respectively. Data are plotted as mean $\pm \mathrm{SEM}\left(\mathrm{c}, \mathrm{c}^{\prime}\right)$, median with

930 interquartile range (e) or percentage of embryos (f) from two (c,c'), or three (e, f) batches of embryos. One-way ANOVA followed by Tukey's post hoc test (c), unpaired t-test with Welch's correction (c') non-parametric Kruskal-Wallis test followed by

Dunn's post hoc test (e) and Fisher's exact test (f) are used to assess statistical

934 significance (ns $=$ not significant, ${ }^{* *} p<0.01$, ${ }^{* * *} p<0.001,{ }^{* * *} p<0.0001$ ). Multiplicity

935 adjusted $P$ values are reported for (c, c') and (e). 
a

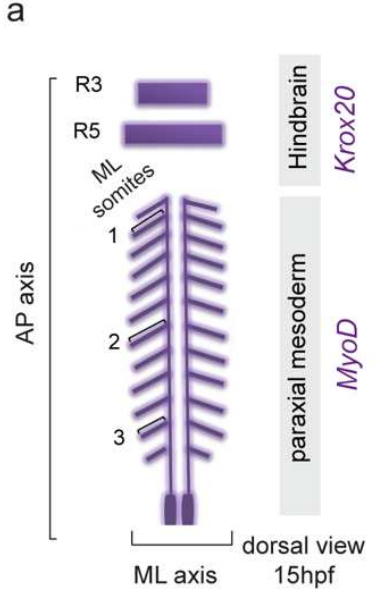

b

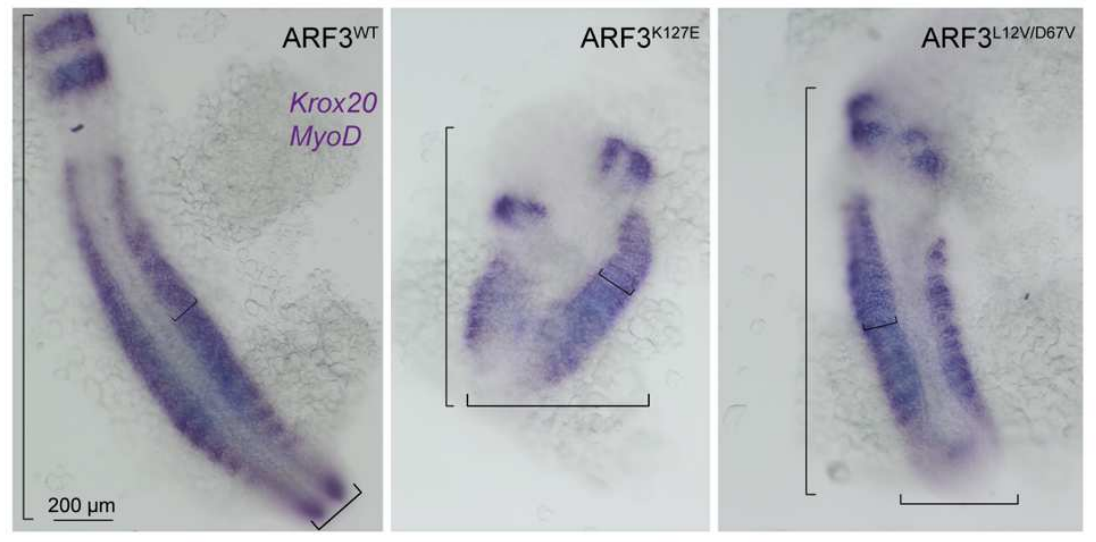

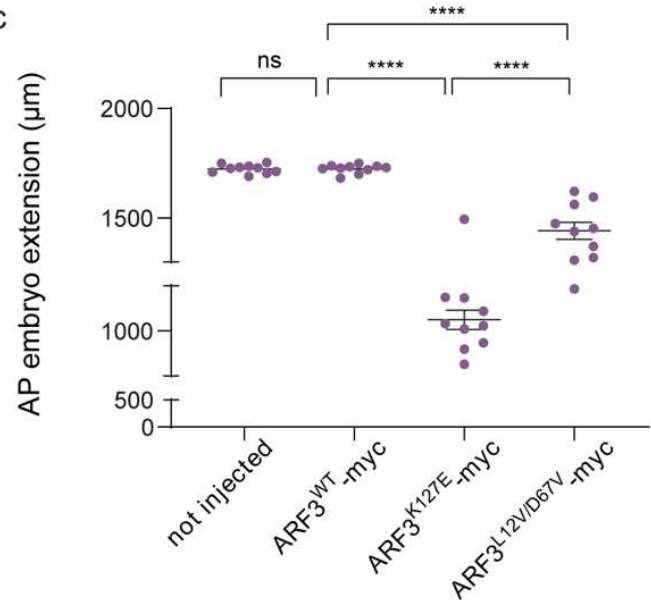

e

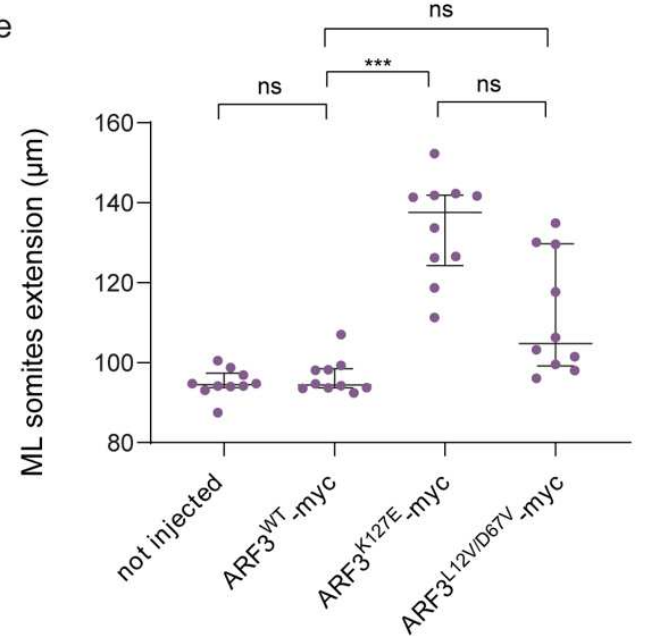

d

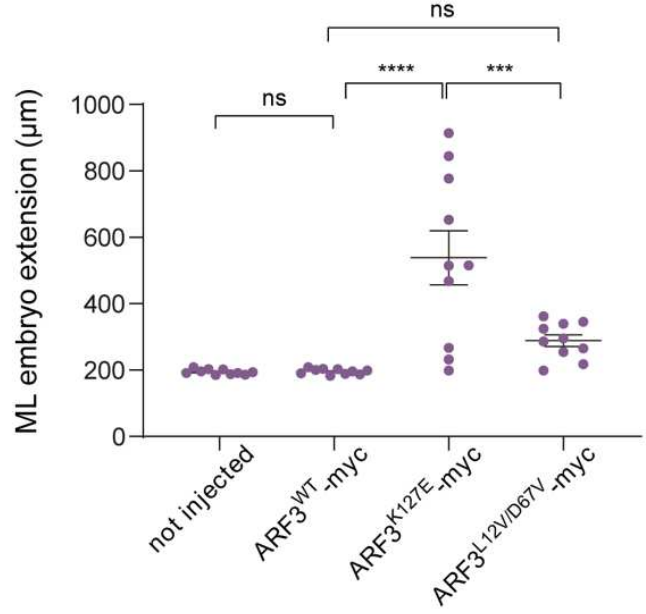

f

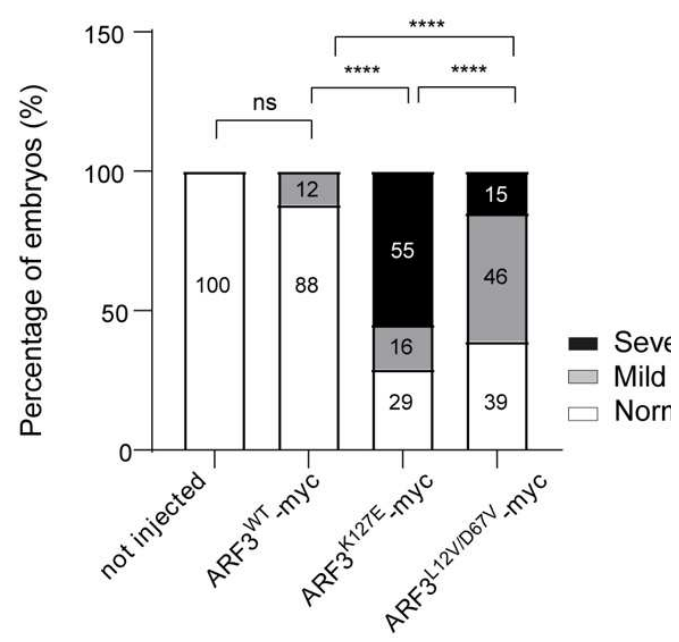

937 Figure 5. Zebrafish embryos expressing ARF3 mutant proteins exhibit defective

938 axes formation during segmentation. (a) Schematic representation of Krox20 and 
Dominantly acting variants in ARF3 have disruptive consequences on Golgi integrity and cause microcephaly recapitulated in zebrafish

Fasano, Muto, Radio, et al.

939

940

941

942

943

944

945

946

947

948

949

950

951

952

953

954

955

956

957

958

959

960

MyoD mRNA localization (light violet), labeling rhombomeres 3 and 5 (hindbrain) and developing somites (in the paraxial mesoderm), respectively, at $15 \mathrm{hpf}$. Whole-mount in situ hybridization is used to measure $\mathrm{AP}$ and $\mathrm{ML}$ embryo extension and $\mathrm{ML}$ extension of somites $(1,2,3$ indicate the anterior, medial and posterior somites, respectively). (b) Representative bright field images showing Krox20 and MyoD in situ mRNA staining in fish expressing WT and mutant ARF3, aberrant AP and ML axes and somite width are indicated by black square brackets, scale bar $=200 \mu \mathrm{m}$. (c-e) AP embryo extension is reduced (c) while ML embryo (d) and somite (e) extension are increased in fish expressing ARF3 mutants, $n=10$. (f) Frequency of $A P$ and $M L$ extension defects, $\mathrm{n}=44,48,37$ and 33 for not injected, $\mathrm{ARF}^{\mathrm{WT}}$, ARF3 ${ }^{\mathrm{K} 127 \mathrm{E}}$ and $A R F 3^{L 12 V ; D 67 V}$, respectively. Unaffected embryos were characterized by an unaltered MyoD and Krox20 mRNA expression compared to not-injected controls, while the mildly and severely affected embryos showed a reduced expression of the two markers along the AP axis and expansion of the ML axis. Severe cases also showed pronounced ML convergence defects, which were evident by the widely separated left and right MyoD-positive domains. Data are plotted as mean + SEM (c, d), median with interquartile range (e) or percentage of the embryos (f) from two (c-e) and three (f) batches of embryos. One-way Anova with Tukey's post hoc test (c, d), non-parametric Kruskal-Wallis with Dunn's post hoc test (e) or Fisher's exact test in a $2 \times 2$ contingency table (f; severe vs. normal) (ns= not significant, $\left.{ }^{*} p<0.05,{ }^{* * *} p<0.001,{ }^{* * * *} p<0.0001\right)$ are used to assess statistical significance. For $\mathrm{c}, \mathrm{d}$ and e the multiplicity adjusted $\mathrm{P}$ values are reported. 
Dominantly acting variants in ARF3 have disruptive consequences on Golgi integrity and cause microcephaly recapitulated in zebrafish

Fasano, Muto, Radio, et al.

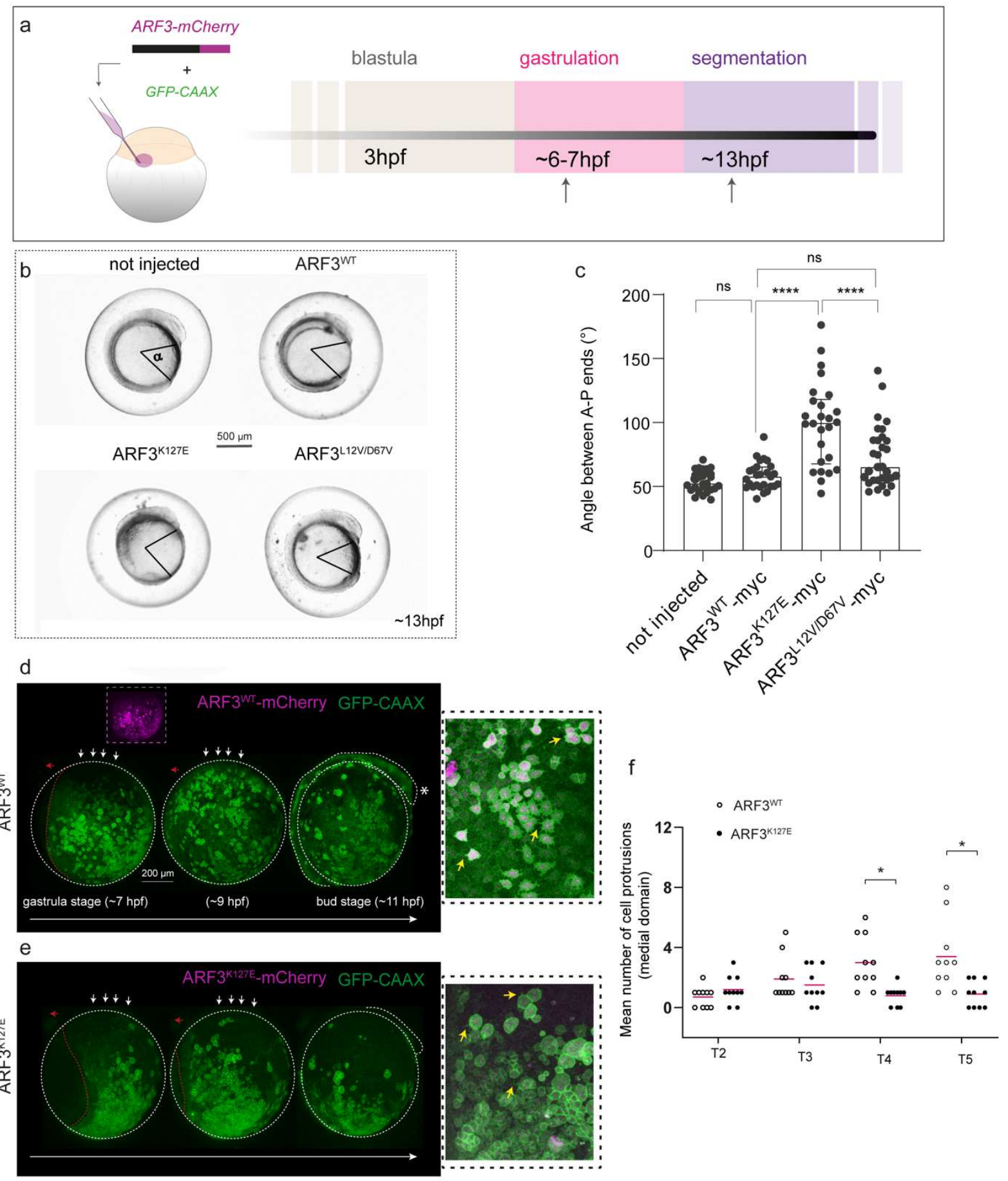

962 Figure 6. Overexpression of activating ARF3 ${ }^{\mathrm{K} 127 \mathrm{E}}$ in zebrafish embryos impairs convergence and extension movements during gastrulation. (a) Schematics of the experimental design. mCherry-tagged ARF3 and GFP-tagged CAAX are injected in one-cell stage embryos and morphometric measurements are performed between 6 
Dominantly acting variants in ARF3 have disruptive consequences on Golgi integrity and cause microcephaly recapitulated in zebrafish

Fasano, Muto, Radio, et al.

966

967

hpf (gastrulation) and $13 \mathrm{hpf}$ (segmentation). (b) Representative bright field images from live embryos injected with $\mathrm{ARF} 3^{\mathrm{WT}}$ and ARF3 mutant mRNAs (K127E and $\mathrm{L} 12 \mathrm{~V} ; \mathrm{D} 67 \mathrm{~V})$ at $13 \mathrm{hpf}$. Lateral view with anterior region on the top is shown. The angle (alpha, black) between the anterior end and posterior end anlage, reflecting the extent of antero-posterior (AP) axis extension, is shown. (c) Values of the angle alpha showing the extension of the AP axis and measured in $A R F 3^{W T}$ and $A R F 3^{K 127 E}$ and $A R F 3^{L 12 V ; D 67 V}$ embryos are shown, with a significant increase recorded in $A R F 3^{K 127 E}$ mutants, $n=30,29,26,31$ for not injected, ARF3 ${ }^{W T}$, ARF3 ${ }^{\mathrm{K} 127 E}$ and ARF3 ${ }^{L 12 V ; D 67 V}$, respectively. (d-e) Confocal images (maximum z-projections) from a live time-lapse experiment showing lateral view of the embryos at different time points during gastrulation. Images show cells labelled with GFP-tagged CAAX protein expression labeling cellular outlines. Expression of mCherry-tagged ARF3 ${ }^{W T}$ in the injected embryos is shown in the upper inset. Higher magnifications on the right (black dashed boxes) show cell protrusions (some are indicated with yellow arrows) of medial cells at circa $8 \mathrm{hpf}$ in $\mathrm{ARF} 3^{\mathrm{WT}}$ and ARF3 ${ }^{\mathrm{K} 127 \mathrm{E}}$ embryos. (f) Number of cell protrusions counted for 10 cells located in the medial portion (centre) of the embryo (between 7 and $8 \mathrm{hpf}$ ), $n=1$. Data are plotted as median with interquartile range from two batches of embryos (c) and mean value of the cells (f). Nonparametric Kruskal-Wallis with Dunn's post hoc test (c) or Two-way Anova, with mixed-effects model analysis and Sidak's post hoc test (f) $\left({ }^{*} p<0.05,{ }^{* *} p<0.01 ;{ }^{* * * *} p<0.0001\right)$ are used to assess statistical significance. For $\mathrm{c}$ the multiplicity adjusted $\mathrm{P}$ values are reported 
Dominantly acting variants in ARF3 have disruptive consequences on Golgi integrity and cause microcephaly recapitulated in zebrafish

Fasano, Muto, Radio, et al.

\section{References}

1. D'Souza-Schorey, C. \& Chavrier, P. ARF proteins: roles in membrane traffic and beyond. Nat. Rev. Mol. Cell Biol. 7, 347-358 (2006).

2. Cockcroft, S. et al. Phospholipase D: a downstream effector of ARF in granulocytes. Science 263, 523-526 (1994).

3. Guo, Y., Sirkis, D. W. \& Schekman, R. Protein Sorting at the trans-Golgi Network. Annu. Rev. Cell Dev. Biol. 30, 169-206 (2014).

4. Makhoul, C., Gosavi, P. \& Gleeson, P. A. Golgi Dynamics: The Morphology of the Mammalian Golgi Apparatus in Health and Disease. Front. Cell Dev. Biol. 7, 112 (2019).

5. Wei, J.-H. \& Seemann, J. Golgi ribbon disassembly during mitosis, differentiation and disease progression. Curr. Opin. Cell Biol. 47, 43-51 (2017).

6. Seifert, W. et al. Cohen syndrome-associated protein, $\mathrm{COH} 1$, is a novel, giant Golgi matrix protein required for Golgi integrity. J. Biol. Chem. 286, 37665-37675 (2011).

7. Shamseldin, H. E., Bennett, A. H., Alfadhel, M., Gupta, V. \& Alkuraya, F. S. GOLGA2, encoding a master regulator of golgi apparatus, is mutated in a patient with a neuromuscular disorder. Hum. Genet. 135, 245-251 (2016).

8. Rasika, S., Passemard, S., Verloes, A., Gressens, P. \& El Ghouzzi, V. Golgipathies in Neurodevelopment: A New View of Old Defects. Dev. Neurosci. 40, 396-416 (2018).

9. Dupuis, N. et al. Dymeclin deficiency causes postnatal microcephaly, hypomyelination and reticulum-to-Golgi trafficking defects in mice and humans. Hum. Mol. Genet. 24, 2771-2783 (2015). 
Dominantly acting variants in ARF3 have disruptive consequences on Golgi integrity and cause microcephaly recapitulated in zebrafish

Fasano, Muto, Radio, et al.

10. Chavrier, P. \& Goud, B. The role of ARF and Rab GTPases in membrane transport. Curr. Opin. Cell Biol. 11, 466-475 (1999).

11. Volpicelli-Daley, L. A., Li, Y., Zhang, C.-J. \& Kahn, R. A. Isoform-selective Effects of the Depletion of ADP-Ribosylation Factors 1-5 on Membrane Traffic. Mol. Biol. Cell 16, 4495-4508 (2005).

12. Donaldson, J. G. \& Jackson, C. L. ARF family G proteins and their regulators: roles in membrane transport, development and disease. Nat. Rev. Mol. Cell Biol. 12, 362375 (2011).

13. Zhang, C. J. et al. Expression of a dominant allele of human ARF1 inhibits membrane traffic in vivo. J. Cell Biol. 124, 289-300 (1994).

14. Gillingham, A. K. \& Munro, S. The small G proteins of the Arf family and their regulators. Annu. Rev. Cell Dev. Biol. 23, 579-611 (2007).

15. Amor, J. C., Harrison, D. H., Kahn, R. A. \& Ringe, D. Structure of the human ADPribosylation factor 1 complexed with GDP. Nature 372, 704-708 (1994).

16. Kjeldgaard, M., Nyborg, J. \& Clark, B. F. The GTP binding motif: variations on a theme. FASEB J. Off. Publ. Fed. Am. Soc. Exp. Biol. 10, 1347-1368 (1996).

17. Goldberg, J. Structural basis for activation of ARF GTPase: mechanisms of guanine nucleotide exchange and GTP-myristoyl switching. Cell 95, 237-248 (1998).

18. Donaldson, J. G., Finazzi, D. \& Klausner, R. D. Brefeldin A inhibits Golgi membranecatalysed exchange of guanine nucleotide onto ARF protein. Nature 360, 350-352 (1992).

19. Helms, J. B. \& Rothman, J. E. Inhibition by brefeldin A of a Golgi membrane enzyme that catalyses exchange of guanine nucleotide bound to ARF. Nature 360, 352-354 (1992). 
Dominantly acting variants in ARF3 have disruptive consequences on Golgi integrity and cause microcephaly recapitulated in zebrafish

Fasano, Muto, Radio, et al.

20. Randazzo, P. A., Yang, Y. C., Rulka, C. \& Kahn, R. A. Activation of ADP-ribosylation factor by Golgi membranes. Evidence for a brefeldin A- and protease-sensitive activating factor on Golgi membranes. J. Biol. Chem. 268, 9555-9563 (1993).

21. Antonny, B., Huber, I., Paris, S., Chabre, M. \& Cassel, D. Activation of ADPribosylation factor 1 GTPase-activating protein by phosphatidylcholine-derived diacylglycerols. J. Biol. Chem. 272, 30848-30851 (1997).

22. Randazzo, P. A. Resolution of two ADP-ribosylation factor 1 GTPase-activating proteins from rat liver. Biochem. J. 324 ( Pt 2), 413-419 (1997).

23. Reinhard, C., Schweikert, M., Wieland, F. T. \& Nickel, W. Functional reconstitution of COPI coat assembly and disassembly using chemically defined components. Proc. Natl. Acad. Sci. U. S. A. 100, 8253-8257 (2003).

24. Gilbert, C. E., Sztul, E. \& Machamer, C. E. Commonly used trafficking blocks disrupt ARF1 activation and the localization and function of specific Golgi proteins. Mol. Biol. Cell 29, 937-947 (2018).

25. Munro, S. The golgin coiled-coil proteins of the Golgi apparatus. Cold Spring Harb. Perspect. Biol. 3, a005256 (2011).

26. Kulkarni-Gosavi, P., Makhoul, C. \& Gleeson, P. A. Form and function of the Golgi apparatus: scaffolds, cytoskeleton and signalling. FEBS Lett. 593, 2289-2305 (2019).

27. Kondo, Y. et al. ARF1 and ARF3 are required for the integrity of recycling endosomes and the recycling pathway. Cell Struct. Funct. 37, 141-154 (2012).

28. Dascher, C. \& Balch, W. E. Dominant inhibitory mutants of ARF1 block endoplasmic reticulum to Golgi transport and trigger disassembly of the Golgi apparatus. J. Biol. Chem. 269, 1437-1448 (1994). 
Dominantly acting variants in ARF3 have disruptive consequences on Golgi integrity and cause microcephaly recapitulated in zebrafish

Fasano, Muto, Radio, et al.

29. Altan-Bonnet, N., Phair, R. D., Polishchuk, R. S., Weigert, R. \& Lippincott-Schwartz, J. A role for Arf1 in mitotic Golgi disassembly, chromosome segregation, and cytokinesis. Proc. Natl. Acad. Sci. U. S. A. 100, 13314-13319 (2003).

30. Hanai, A. et al. Class I Arfs (Arf1 and Arf3) and Arf6 are localized to the Flemming body and play important roles in cytokinesis. J. Biochem. (Tokyo) 159, 201-208 (2016).

31. Nakayama, K. Regulation of cytokinesis by membrane trafficking involving small GTPases and the ESCRT machinery. Crit. Rev. Biochem. Mol. Biol. 51, 1-6 (2016).

32. Rodrigues, F. F. \& Harris, T. J. C. Key roles of Arf small G proteins and biosynthetic trafficking for animal development. Small GTPases 10, 403-410 (2019).

33. Carvajal-Gonzalez, J. M. et al. The clathrin adaptor AP-1 complex and Arf1 regulate planar cell polarity in vivo. Nat. Commun. 6, 6751 (2015).

34. Ge, X. et al. Missense-depleted regions in population exomes implicate ras superfamily nucleotide-binding protein alteration in patients with brain malformation. NPJ Genomic Med. 1, (2016).

35. Sobreira, N., Schiettecatte, F., Valle, D. \& Hamosh, A. GeneMatcher: a matching tool for connecting investigators with an interest in the same gene. Hum. Mutat. 36, 928930 (2015).

36. Denayer, E. et al. Mutation analysis in Costello syndrome: functional and structural characterization of the HRAS p.Lys117Arg mutation. Hum. Mutat. 29, 232-239 (2008).

37. Lim, Y. H. et al. Somatic Activating RAS Mutations Cause Vascular Tumors Including Pyogenic Granuloma. J. Invest. Dermatol. 135, 1698-1700 (2015).

38. Schubbert, S. et al. Germline KRAS mutations cause Noonan syndrome. Nat. Genet. 38, 331-336 (2006). 
Dominantly acting variants in ARF3 have disruptive consequences on Golgi integrity and cause microcephaly recapitulated in zebrafish

Fasano, Muto, Radio, et al.

39. Zenker, M. et al. Expansion of the genotypic and phenotypic spectrum in patients with KRAS germline mutations. J. Med. Genet. 44, 131-135 (2007).

40. Landrum, M. J. et al. ClinVar: improving access to variant interpretations and supporting evidence. Nucleic Acids Res. 46, D1062-D1067 (2018).

41. Nava, C. et al. Cardio-facio-cutaneous and Noonan syndromes due to mutations in the RAS/MAPK signalling pathway: genotype-phenotype relationships and overlap with Costello syndrome. J. Med. Genet. 44, 763-771 (2007).

42. Gripp, K. W. \& Lin, A. E. Costello syndrome: a Ras/mitogen activated protein kinase pathway syndrome (rasopathy) resulting from HRAS germline mutations. Genet.

Med. Off. J. Am. Coll. Med. Genet. 14, 285-292 (2012).

43. Lee, Y. et al. Makes caterpillars floppy-like effector-containing MARTX toxins require host ADP-ribosylation factor (ARF) proteins for systemic pathogenicity. Proc. Natl. Acad. Sci. U. S. A. 116, 18031-18040 (2019).

44. Carta, C. et al. Germline missense mutations affecting KRAS Isoform B are associated with a severe Noonan syndrome phenotype. Am. J. Hum. Genet. 79, 129-135 (2006).

45. Zampino, G. et al. Diversity, parental germline origin, and phenotypic spectrum of de novo HRAS missense changes in Costello syndrome. Hum. Mutat. 28, 265-272 (2007).

46. Pasqualato, S., Renault, L. \& Cherfils, J. Arf, Arl, Arp and Sar proteins: a family of GTP-binding proteins with a structural device for 'front-back' communication. EMBO Rep. 3, 1035-1041 (2002).

47. Ménétrey, J. et al. Structural basis for ARF1-mediated recruitment of ARHGAP21 to Golgi membranes. EMBO J. 26, 1953-1962 (2007). 
Dominantly acting variants in ARF3 have disruptive consequences on Golgi integrity and cause microcephaly recapitulated in zebrafish

Fasano, Muto, Radio, et al.

48. Yu, X., Breitman, M. \& Goldberg, J. A Structure-Based Mechanism for Arf1Dependent Recruitment of Coatomer to Membranes. Cell 148, 530-542 (2012).

49. Ratcliffe, C. D. H., Sahgal, P., Parachoniak, C. A., Ivaska, J. \& Park, M. Regulation of Cell Migration and $\beta 1$ Integrin Trafficking by the Endosomal Adaptor GGA3. Traffic Cph. Den. 17, 670-688 (2016).

50. Adolf, F. et al. Proteomic Profiling of Mammalian COPII and COPI Vesicles. Cell Rep. 26, 250-265.e5 (2019).

51. Xiang, Y., Seemann, J., Bisel, B., Punthambaker, S. \& Wang, Y. Active ADPribosylation factor-1 (ARF1) is required for mitotic Golgi fragmentation. J. Biol. Chem. 282, 21829-21837 (2007).

52. Manolea, F. et al. Arf3 is activated uniquely at the trans-Golgi network by brefeldin Ainhibited guanine nucleotide exchange factors. Mol. Biol. Cell 21, 1836-1849 (2010).

53. Kimmel, C. B., Ballard, W. W., Kimmel, S. R., Ullmann, B. \& Schilling, T. F. Stages of embryonic development of the zebrafish. Dev. Dyn. Off. Publ. Am. Assoc. Anat. 203, 253-310 (1995).

54. Bagwell, J. et al. Notochord vacuoles absorb compressive bone growth during zebrafish spine formation. eLife 9, (2020).

55. Jessen, J. R. et al. Zebrafish trilobite identifies new roles for Strabismus in gastrulation and neuronal movements. Nat. Cell Biol. 4, 610-615 (2002).

56. Roszko, I., Sawada, A. \& Solnica-Krezel, L. Regulation of convergence and extension movements during vertebrate gastrulation by the Wnt/PCP pathway. Semin. Cell Dev. Biol. 20, 986-997 (2009).

57. Wallingford, J. B., Fraser, S. E. \& Harland, R. M. Convergent extension: the molecular control of polarized cell movement during embryonic development. Dev. Cell 2, 695-706 (2002). 
Dominantly acting variants in ARF3 have disruptive consequences on Golgi integrity and cause microcephaly recapitulated in zebrafish

Fasano, Muto, Radio, et al.

58. Tate, J. G. et al. COSMIC: the Catalogue Of Somatic Mutations In Cancer. Nucleic Acids Res. 47, D941-D947 (2019).

59. Tartaglia, M. \& Gelb, B. D. Disorders of dysregulated signal traffic through the RASMAPK pathway: phenotypic spectrum and molecular mechanisms. Ann. N. Y. Acad. Sci. 1214, 99-121 (2010).

60. Motta, M. et al. Activating MRAS mutations cause Noonan syndrome associated with hypertrophic cardiomyopathy. Hum. Mol. Genet. 29, 1772-1783 (2020).

61. Flex, E. et al. Activating mutations in RRAS underlie a phenotype within the RASopathy spectrum and contribute to leukaemogenesis. Hum. Mol. Genet. 23, 4315-4327 (2014).

62. Niihori, T. et al. Germline-Activating RRAS2 Mutations Cause Noonan Syndrome. Am. J. Hum. Genet. 104, 1233-1240 (2019).

63. Capri, Y. et al. Activating Mutations of RRAS2 Are a Rare Cause of Noonan Syndrome. Am. J. Hum. Genet. 104, 1223-1232 (2019).

64. Khan, A. R. \& Ménétrey, J. Structural biology of Arf and Rab GTPases' effector recruitment and specificity. Struct. Lond. Engl. 1993 21, 1284-1297 (2013).

65. Merithew, E. et al. Structural Plasticity of an Invariant Hydrophobic Triad in the Switch Regions of Rab GTPases Is a Determinant of Effector Recognition*. J. Biol. Chem. 276, 13982-13988 (2001).

66. Kuai, J. \& Kahn, R. A. Residues forming a hydrophobic pocket in ARF3 are determinants of GDP dissociation and effector interactions. FEBS Lett. 487, 252-256 (2000).

67. Banne, E. et al. West syndrome, microcephaly, grey matter heterotopia and hypoplasia of corpus callosum due to a novel ARFGEF2 mutation. J. Med. Genet. 50, 772-775 (2013). 
Dominantly acting variants in ARF3 have disruptive consequences on Golgi integrity and cause microcephaly recapitulated in zebrafish

Fasano, Muto, Radio, et al.

68. Horton, A. C. et al. Polarized Secretory Trafficking Directs Cargo for Asymmetric Dendrite Growth and Morphogenesis. Neuron 48, 757-771 (2005).

69. Lauri, A., Fasano, G., Venditti, M., Dallapiccola, B. \& Tartaglia, M. In vivo functional genomics for undiagnosed patients: the impact of small GTPases signaling dysregulation at pan-embryo developmental scale. Front. Cell Dev. Biol. 9, (2021).

70. Prigozhina, N. L. \& Waterman-Storer, C. M. Decreased polarity and increased random motility in PtK1 epithelial cells correlate with inhibition of endosomal recycling. J. Cell Sci. 119, 3571-3582 (2006).

71. Winter, J. F. et al. Caenorhabditis elegans screen reveals role of PAR-5 in RAB-11recycling endosome positioning and apicobasal cell polarity. Nat. Cell Biol. 14, 666676 (2012).

72. Ulrich, F. et al. Wnt11 Functions in Gastrulation by Controlling Cell Cohesion through Rab5c and E-Cadherin. Dev. Cell 9, 555-564 (2005).

73. Sarmah, S. et al. Sec24D-dependent transport of extracellular matrix proteins is required for zebrafish skeletal morphogenesis. PloS One 5, e10367 (2010).

74. Coutinho, P. et al. Differential requirements for COPI transport during vertebrate early development. Dev. Cell 7, 547-558 (2004).

75. Sheen, V. L. et al. Mutations in ARFGEF2 implicate vesicle trafficking in neural progenitor proliferation and migration in the human cerebral cortex. Nat. Genet. 36, 69-76 (2004).

76. Pezzani, L. et al. Atypical presentation of pediatric BRAF RASopathy with acute encephalopathy. Am. J. Med. Genet. A. 176, 2867-2871 (2018).

77. Ziegler, A. et al. Confirmation that variants in TTI2 are responsible for autosomal recessive intellectual disability. Clin. Genet. 96, 354-358 (2019). 
Dominantly acting variants in ARF3 have disruptive consequences on Golgi integrity and cause microcephaly recapitulated in zebrafish

Fasano, Muto, Radio, et al.

78. Radio, F. C. et al. SPEN haploinsufficiency causes a neurodevelopmental disorder overlapping proximal 1p36 deletion syndrome with an episignature of $X$ chromosomes in females. Am. J. Hum. Genet. 108, 502-516 (2021).

79. Lin, Y.-C. et al. SCUBE3 loss-of-function causes a recognizable recessive developmental disorder due to defective bone morphogenetic protein signaling. Am. J. Hum. Genet. 108, 115-133 (2021).

80. Vetro, A. et al. ATP1A2- and ATP1A3-associated early profound epileptic encephalopathy and polymicrogyria. Brain J. Neurol. (2021) doi:10.1093/brain/awab052.

81. Humphrey, W., Dalke, A. \& Schulten, K. VMD: visual molecular dynamics. J. Mol. Graph. 14, 33-38, 27-28 (1996).

82. Waterhouse, A. et al. SWISS-MODEL: homology modelling of protein structures and complexes. Nucleic Acids Res. 46, W296-W303 (2018).

83. Pettersen, E. F. et al. UCSF Chimera--a visualization system for exploratory research and analysis. J. Comput. Chem. 25, 1605-1612 (2004).

84. Dunbrack, R. L. Rotamer libraries in the 21st century. Curr. Opin. Struct. Biol. 12, 431-440 (2002).

85. Páll, S. et al. Heterogeneous parallelization and acceleration of molecular dynamics simulations in GROMACS. J. Chem. Phys. 153, 134110 (2020).

86. Huang, J. \& MacKerell, A. D. CHARMM36 all-atom additive protein force field: validation based on comparison to NMR data. J. Comput. Chem. 34, 2135-2145 (2013).

87. Bussi, G., Donadio, D. \& Parrinello, M. Canonical sampling through velocity rescaling. J. Chem. Phys. 126, 014101 (2007). 
Dominantly acting variants in ARF3 have disruptive consequences on Golgi integrity and cause microcephaly recapitulated in zebrafish

Fasano, Muto, Radio, et al.

88. Darden, T., York, D. \& Pedersen, L. Particle mesh Ewald: An N·log(N) method for Ewald sums in large systems. J. Chem. Phys. 98, 10089-10092 (1993).

89. Villefranc, J. A., Amigo, J. \& Lawson, N. D. Gateway compatible vectors for analysis of gene function in the zebrafish. Dev. Dyn. Off. Publ. Am. Assoc. Anat. 236, 30773087 (2007).

90. Don, E. K. et al. A Tol2 Gateway-Compatible Toolbox for the Study of the Nervous System and Neurodegenerative Disease. Zebrafish 14, 69-72 (2017).

91. Cole, N. B. et al. Diffusional mobility of Golgi proteins in membranes of living cells. Science 273, 797-801 (1996).

92. Makyio, H. et al. Structural basis for Arf6-MKLP1 complex formation on the Flemming body responsible for cytokinesis. EMBO J. 31, 2590-2603 (2012).

93. LaFave, M. C., Varshney, G. K., Vemulapalli, M., Mullikin, J. C. \& Burgess, S. M. A defined zebrafish line for high-throughput genetics and genomics: NHGRI-1. Genetics 198, 167-170 (2014).

94. Peri, F. \& Nüsslein-Volhard, C. Live Imaging of Neuronal Degradation by Microglia Reveals a Role for v0-ATPase a1 in Phagosomal Fusion In Vivo. Cell 133, 916-927 (2008).

95. Westerfield, M. The zebrafish book. A guide for the laboratory use of zebrafish (Danio rerio. (2000).

96. Kovach, W. L. Oriana - Circular Statistics for Windows. (2011).

97. Thisse, C. \& Thisse, B. High-resolution in situ hybridization to whole-mount zebrafish embryos. Nat. Protoc. 3, 59-69 (2008).

98. Schindelin, J. et al. Fiji: an open-source platform for biological-image analysis. Nat. Methods 9, 676-682 (2012). 


\section{Supplementary Files}

This is a list of supplementary files associated with this preprint. Click to download.

- FasanoMutoRadioTable1.pdf

- SupplementaryInformationFasanoMutoRadioetal20210107021.pdf

- SupplementaryVideo1.mp4 